\title{
The lncRNA SEMA3B-AS1/HMGB1/FBXW7 Axis Mediates the Peritoneal Metastasis of Gastric Cancer by Regulating BGN Protein Ubiquitination
}

\author{
Guoquan Huang, ${ }^{1,2,3}$ Zhenxian Xiang, ${ }^{1,3}$ Haitao Wu, ${ }^{1,3}$ Qiuming He, ${ }^{1,3}$ Rongzhang Dou, ${ }^{1,3}$ \\ Chaogang Yang, ${ }^{1,3}$ Jialin Song, ${ }^{1,3}$ Sihao Huang, ${ }^{1,3}$ Shuyi Wang $\mathbb{D}^{1,3}$ and Bin Xiong $\mathbb{D}^{1,3}$ \\ ${ }^{1}$ Department of Gastrointestinal Surgery \& Department of Gastric and Colorectal Surgical Oncology, Zhongnan Hospital of \\ Wuhan University, Wuhan, China \\ ${ }^{2}$ Department of Gastrointestinal Surgery, Central Hospital of Enshi Tujia and Miao Autonomous Prefecture, Enshi, China \\ ${ }^{3}$ Hubei Cancer Clinical Study Center, Wuhan, China
}

Correspondence should be addressed to Shuyi Wang; shuyiwang@whu.edu.cn and Bin Xiong; binxiong1961@whu.edu.cn

Received 30 October 2021; Accepted 27 December 2021; Published 27 February 2022

Academic Editor: Laura Bravo

Copyright (c) 2022 Guoquan Huang et al. This is an open access article distributed under the Creative Commons Attribution License, which permits unrestricted use, distribution, and reproduction in any medium, provided the original work is properly cited.

\begin{abstract}
Peritoneal metastasis (PM) is one of the main causes of a poor prognosis in patients with advanced gastric cancer (GC). lncRNAs have been confirmed to play a very crucial role in the occurrence, development, and metastasis of many human cancers, including gastric cancer. However, the mechanism of lncRNA in PM of GC is rarely studied. We explored the mechanism of PM of GC through lncRNA gene sequencing and protein profiling analysis to detect PM-associated lncRNAs and proteins. A quantitative reverse transcription polymerase chain reaction (qRT-PCR) was performed to identify the mRNA expression of SEMA3B-AS1 and BGN in GC tissues and adjacent normal tissues. The biological function of SEMA3B-AS1 in the PM of GC was identified through gain- and loss-of-function assays. Chromatin isolation by RNA purification (ChIRP), RNA immunoprecipitation (RIP), RNA pull-down, luciferase reporter, and coimmunoprecipitation (co-IP) assays was carried out to demonstrate the potential mechanism between SEMA3B-AS1 and its downstream genes, including HMGB1, FBXW7, and BGN. Finally, the biological function of SEMA3B-AS1 was demonstrated in animal experiments. The mRNA expression level of SEMA3B-AS1 was downregulated in GC and PM tissues compared to normal stomach tissues; however, BGN was highly expressed at the mRNA level. SEMA3B-AS1 was closely related to PM and the overall survival (OS) of GC patients. Functionally, the overexpression of SEMA3B-AS1 was related to GC progression, PM, and prognosis. Mechanistically, SEMA3B-AS1 could combine with HMGB1 to regulate the transcription of FBXW7, thus facilitating the ubiquitination of BGN. In conclusion, our study demonstrated that the SEMA3B-AS1/HMGB1/FBXW7 axis plays an inhibitory role in the PM of GC by regulating BGN protein ubiquitination. It also provides a new biological marker for the diagnosis and treatment of the PM of GC.
\end{abstract}

\section{Introduction}

Gastric cancer (GC) is one of the leading causes of cancerrelated death worldwide $[1,2]$. Although great progress has been made in the treatment of GC, the prognosis of the peritoneal metastasis (PM) of GC remains poor [3, 4]. Recent studies have demonstrated that PM accounts for approximately $53 \%-66 \%$ of cases of GC distant metastasis [5-7]. At present, the mechanism of the PM of GC remains unclear, and there is a lack of effective means for the early diagnosis and treatment of PM. Therefore, it is urgent for us to further explore the molecular biological mechanism of the PM of GC and find new diagnostic and therapeutic markers.

Long noncoding RNAs (lncRNAs) play a vital role in the occurrence, development, invasion, and metastasis of cancer. lncRNAs are more than $200 \mathrm{nt}$ in length and have a conserved secondary structure, and most of them do not have 
protein-coding function [8]. Existing evidence demonstrates that $\operatorname{lncRNAs}$ have crucial biological functions. They can interact with proteins, DNA, and RNA and participate in the regulation of a variety of biological processes $[9,10]$. They can also regulate gene expression through various mechanisms, including epigenetic regulation, chromatin modification, transcription, posttranscriptional regulation, and protein metabolism [11-13]. Huang et al. found that lncRNAs can interact with cancer stem cells and then induce cancer metastasis and recurrence [14]. At present, the role of lncRNA in PM of GC remains to be further explored. According to the lncRNA sequencing in primary GC tissues and GC PM tissues, SEMA3B-AS1 was one of the most downregulated lncRNAs in PM tissues. Mass spectrometry (MS) analysis of SEMA3B-AS1 pull-down further revealed that mobility group box 1 (HMGB1) was an interacting protein of SEMA3B-AS1. Evidence demonstrated that HMGB1 is a multifunctional molecule in cell homeostasis. In the nucleus, HMGB1 plays an important protective role in the process of DNA replication, transcription, and chromatin remodeling, thus regulating DNA damage repair and maintaining genome stability as a DNA chaperone [15]. However, HMGB1 could promote the release of cytokines such as IL-6 and IL- 8 by activating MAPK- and MyD88-dependent NF$\mathrm{KB}$ pathways in the extracellular matrix, thus stimulating tumor cell proliferation, angiogenesis, epithelialmesenchymal transition (EMT), invasion, and metastasis [16]. Tumor suppressor genes such as F-box and WD repeat domain-containing 7 (FBXW7), which we have previously studied, are usually inactivated after the occurrence of GC, and the body will issue instructions for gene repair, which allows tumor suppressor-related IncRNAs to recruit and bind genes or proteins that can repair DNA damage [15, 17-19]. Bioinformatics analysis revealed that HMGB1 was one of the transcription factors of FBXW7. Thus, whether SEMA3B-AS1 could combine with HMGB1 to regulate the transcription of FBXW7 remains unknown.

As is known to all, FBXW7 could regulate the ubiquitination of downstream protein [20]. Then, we find that the BGN protein is the ubiquitination substrate of FBXW7 through UbiBrowser website prediction. The BGN protein, which is a key member of the leucine-rich proteoglycan family and is mainly located in the cellular stroma, is localized on the long arm of human X chromosome Xq13-qter [21]. It is linked to a variety of human cancers and associated with the aggressive growth and metastasis of tumors, such as GC and colon cancer [22-24]. To our surprise, MS and bioinformatics analysis found that BGN is upregulated in PM of GC tissues, and its high expression correlates with metastasis and poor prognosis of GC patients. Therefore, whether FBXW7 could regulate the ubiquitination of BGN deserve to be further elucidation.

In current research, we found that SEMA3B-AS1 was downregulated in PM of GC, and its lower expression correlates with PM of GC and poor prognosis of GC patients. Function analysis revealed that SEMA3B-AS1 inhibited proliferation, migration, and invasion of GC. Mechanistically, SEMA3B-AS1 could combine with HMGB1 to regulate the transcription of FBXW7, thus facilitating the ubiquitination of BGN. Our study provides a new biological marker for the clinical diagnosis and treatment of patients with PM.

\section{Methods and Materials}

2.1. Tissue Samples and Patients. Fifty pairs of GC tissues and paracancerous normal tissues were obtained from the specimen bank of the Cancer Institute of Zhongnan Hospital of Wuhan University. All patients were diagnosed with GC according to the American Joint Committee on Cancer (AJCC) tumor staging manual and histopathological assessment [25], and none of them received preoperative radiotherapy or chemotherapy. All patients provided informed consent for the procedures, which were approved by the Internal Review and Ethics Committee of Zhongnan Hospital of Wuhan University (Wuhan, China; Ethical Approval Number: 2019079).

2.2. Immunohistochemistry (IHC) and Immunofluorescence (IF). IHC: the specimens were fixed in $10 \%$ formaldehyde within 30 minutes and then paraffin embedded. Before the immunohistochemistry experiment, $4 \mu \mathrm{m}$ thick serial sections were made with a slicer and fixed on slides. Then, we performed immunohistochemistry experiments according to the manufacturer's protocol. Each specimen was incubated with monoclonal antibodies against human FBXW7 $(1: 200$; Abcam, USA) and BGN ( $1: 200$; CST, USA) overnight at $4^{\circ} \mathrm{C}$. After eluting the primary antibody, the secondary antibody was routinely incubated for 50 minutes. Immunostaining was performed using $\mathrm{DAB}$ reagent, and the cell nucleus was counterstained. Finally, the samples were dehydrated with anhydrous ethanol and sealed with xylene transparent neutral gum. We scanned and analyzed the samples with an automatic digital slide scanning and analysis system (Aperio VERSA 8, Germany) and used the immune response score (IRS) method to semiquantitatively score the gene expression levels [26].

IF: we prepare cell sliders and perform IF experiments according to the merchant's instructions. Antibody against human HMGB1 $(1: 200$, Proteintech, USA) was used. We scanned and analyzed the samples with an automatic digital slide scanning and analysis system (Aperio VERSA 8, Germany).

2.3. Cell Line Culture. The cell lines involved in this study included five GC cell lines (AGS, HGC-27, MGC-803, MKN-45, and SGC-7901) and a human stomach epithelial cell line (GES-1), which were obtained from the Cell Bank of Wuhan University. All cells were incubated in an incubator with $5 \% \mathrm{CO}_{2}$ at $37^{\circ} \mathrm{C}$ using DMEM (Gibco, USA) containing $10 \%$ fetal bovine serum (FBS) (Gibco, USA) and $2 \mathrm{mmol} / \mathrm{L}$ glutamine. When the cell density reached more than $90 \%, 0.25 \%$ trypsin was used to isolate the cells for subculture and subsequent experiments.

2.4. RNA Extraction and Quantitative Reverse Transcription Polymerase Chain Reaction ( $q R T-P C R$ ) Assay. Total RNA was extracted from the GC cell lines and tissue samples by using the EASYspin Tissue and Cellular RNA Rapid Extraction Kit (Aidlab, Beijing, China) or TRIzol Reagent 
(Invitrogen, USA) according to the manufacturer's protocol. Then, the concentration of total RNA was measured by a NanoDrop 2000 full-spectrum spectrophotometer (Thermo Scientific, USA), and $1 \mu \mathrm{g}$ of RNA from each sample was reverse-transcribed into cDNA according to the related kit's instructions (Vazyme, Nanjing, China). Next, we performed qRT-PCR experiments on a Bio-Rad IQ5 real-time PCR instrument (Bio-Rad, USA) by using SYBR-Green PCR Master Mix (Vazyme, Nanjing, China) in a $20 \mu \mathrm{L}$ reaction system. The $2^{-\Delta \Delta \mathrm{Ct}}$ method was used to calculate the relative expression of the target gene. The gene primer sequences involved in this study are shown in Supplementary Table 1. Finally, the histogram was plotted using GraphPad Prism 8.

\subsection{Subcellular Fractionation Assay for Localization Studies} and RNA Fluorescence In Situ Hybridization (FISH) Assay. GC cells $\left(1 \times 10^{7}\right)$ were collected and washed twice with ice-cold PBS. According to the manufacturer's protocol, we carried out nuclear and cytosolic fractionation assays using the Nuclear and Cytoplasmic Extraction Kit. Then, total RNA was extracted from the nuclear fraction and cytoplasmic fraction samples by using the RNA Rapid Extraction Kit (Aidlab, Beijing, China). Finally, the relative expression of SEMA3B-AS1 in the nucleus and cytoplasm was detected by qRT-PCR assay, and U6 and $\beta$-actin were used as nuclear and cytoplasmic reference genes, respectively.

The FISH probe of SEMA3B-AS1 was synthesized by GenePharma (Shanghai, China). The probe sequence is shown in Supplementary Table 1. First, we prepared SGC and HGC cells into cell climbing slides and 4\% paraformaldehyde-fixed SGC and HGC cell slides. Then, FISH experiments were conducted according to the FISH kit protocol (Servicebio, Wuhan). In brief, we treated the cells with protease $\mathrm{K}(20 \mu \mathrm{g} / \mathrm{mL})$ for $1-55$ minutes and washed them with PBS three times. Next, we performed prehybridization, hybridization (probe hybridization solution at a concentration of $5 \mu \mathrm{M})$, and DAPI counterstaining of the nuclei. Finally, we observed the results, captured images under a positive fluorescence microscope (NIKON, Japan), and analyzed the results.

2.6. Lentivirus, Plasmids, siRNAs, and Transfection. The construction, synthesis, sequencing, and identification of the plasmid and lentivirus of SEMA3B-AS1 containing green fluorescent protein (GFP) were completed by GenePharma Biotechnology Co. Ltd. The plasmid and lentivirus of BGN, LV-OE-FBXW7/LV-ON-FBXW7, HMGB1-RNAi, FBXW7RNAi (1, 2, 3), and negative control RNA (si-control) were purchased from GeneChem Chemical Technology Co. Ltd. (Shanghai, China). Double luciferase-associated plasmids and HMGB1 overexpression plasmid were purchased from BIOFAVOR BIOTECH. GC cells (SGC-7901, HGC-27) were transfected using Lipofectamine 2000 (Invitrogen, USA) according to the manufacturer's protocol. At 24 or $48 \mathrm{~h}$ after transfection, the cells were plated for functional assay or harvested for RNA or protein correlation analysis, respectively. To construct stably transfected cell lines, lentivirus and polybrene (final $7 \mu \mathrm{g} / \mathrm{mL}$, Sigma-Aldrich) were added to $25 \%$ of the confluent cells, fresh DMEM containing $10 \%$ FBS was added $16 \mathrm{~h}$ after infection, and the medium was exchanged with medium containing the corresponding antibiotics $48 \mathrm{~h}$ later. Finally, stably transfected cell lines were obtained by further screening with puromycin (Sigma-Aldrich, USA) for subsequent tests.

2.7. Protein Extraction, Western Blotting Assay, and Reagents. Cultured GC cells were collected and washed three times with PBS, and then, RIPA buffer containing protease inhibitor (Thermo Scientific, USA) was added to the cells according to the number of cells. The cell lysates were dissolved on ice for 30 minutes and then ultrasonically crushed and quantified by using BCA assays. The proteins were separated by sodium dodecyl sulfate-polyacrylamide gels (SDSPAGE) and transferred to polyvinylidene fluoride (PVDF) membranes (Millipore, USA). Next, the membranes were blocked with $5 \%$ nonfat milk for $2 \mathrm{~h}$, and the primary antibodies were incubated on a shaker in a refrigerator at $4^{\circ} \mathrm{C}$ overnight. Then, the membranes were washed with TBST, and HRP-conjugated secondary antibodies were added for $1-1.5 \mathrm{~h}$ at room temperature. The target proteins were detected with a Bio-Rad ChemiDoc XRS System. Densitometric analysis was performed by using Bio-Rad Image Lab software. The primary antibodies and dilution multiples involved in this study were as follows: anti-FBXW7 (1:1000, Abcam, USA), anti-BGN (1:1000, CST, USA), anti-HMGB1 (1:1000, Proteintech, USA), anti-H3 (1:1000, CST, USA), and anti-GAPDH (1:5000, Santa Cruz, CA). The secondary antibodies were diluted at $1: 5000$.

2.8. Cell Counting Kit-8 (CCK-8) Assay, Colony Formation Assay, and Wound Healing Assay. To detect the changes in cell viability after overexpression or knockdown of SEMA3B -AS1, we performed a CCK-8 assay according to the manufacturer's instructions (Shanghai, China), detected the absorbance of cells at $0 \mathrm{~h}, 24 \mathrm{~h}, 48 \mathrm{~h}, 72 \mathrm{~h}$, and $96 \mathrm{~h}$, and analyzed the differences in absorbance between each group.

A colony formation experiment was carried out to detect the proliferation ability of cells after SEMA3B-AS1 overexpression or interference. In short, SGC-7901 cells and HGC cells were implanted into a six-well plate at 1000 cells per well. After 14 days of culture in an incubator at $37^{\circ} \mathrm{C}$, the samples were fixed with $4 \%$ paraformaldehyde, stained with $0.5 \%$ crystal violet, and then photographed under a microscope (Olympus-IX73, Japan).

A wound healing assay was performed to evaluate the migration ability of GC cells after SEMA3B-AS1 overexpression or knockdown. GC cells were plated into six-well plates, and when the cell density was approximately 90\%, three wounds were created using a plastic pipette tip on the cell surface, and the cellular debris was washed away with PBS. Next, we added serum-free medium and incubated the cells in an incubator at $37^{\circ} \mathrm{C}$. Photos of the same area were taken again every $24 \mathrm{~h}$. Finally, a microscope (Olympus-IX73, Japan) was utilized to take photographs, and the wound area was measured by ImageJ software (NIH, USA). Each experiment was repeated at least three times. 
2.9. Transwell Migration and Invasion Assay. We used 24well plates ( $8 \mu \mathrm{m}$ pore size; Corning, USA) to detect the migration and invasion ability of GC cells after SEMA3BAS1 overexpression or knockdown. A total of $1.0 \times 10^{5}$ cells were suspended in $500 \mu \mathrm{L}$ serum-free DMEM and added to the upper chamber, while $750 \mu \mathrm{L}$ DMEM containing $10 \%$ FBS was added to the lower chamber. After incubation at $37^{\circ} \mathrm{C}$ for $48 \mathrm{~h}$, we removed the cells in the upper chamber, and cells on the lower surface of the membrane were fixed with $4 \%$ paraformaldehyde and stained with crystal violet $(0.5 \%)$. Finally, five visual fields were selected under a microscope (Olympus-IX73, Japan) to take photos and count cells. All experiments were repeated three times.

2.10. RNA Pull-Down Assay. Based on the gene sequence of SEMA3B-AS1, we designed and synthesized a biotin-labeled full-length RNA probe from RiboBio Co. Ltd. (Guangzhou, China).RNA pull-down assays were performed in SEMA3B-AS1-overexpressing cells (HGC-27) using the RNA-Protein Pull-Down Kit (Thermo 20164, USA). In brief, biotinylated full-length RNA probes were mixed with streptavidin agarose beads and incubated overnight at $4^{\circ} \mathrm{C}$. A freshly prepared reagent was then added to each reaction system with RNase inhibitor and protease/phosphatase inhibitor. The mixture was incubated with rotation for $2 \mathrm{~h}$ at room temperature. After washing thoroughly three times, the RNA-protein binding mixture was eluted, and the eluted products were identified through Western blotting or mass spectrometry (MS).

2.11. RNA-Binding Protein Immunoprecipitation (RIP) Assay. To explore the correlation between lncRNAs and proteins, we performed a RIP assay. GC cells (HGC-27 or SGC7901) were cultured and treated accordingly. Next, the cells were harvested and lysed with RIP lysis buffer. Then, we carried out the following experiment according to the protocol of the Magna RIP Kit (Millipore, Billerica, MA, USA). AntiHMGB1 $(5 \mu \mathrm{g}$, Proteintech, USA) and anti-IgG $(2 \mu \mathrm{g})$ antibodies were added and incubated at room temperature for 30 minutes, and total RNA ( $10 \mu \mathrm{L}$, input control) was utilized as a control. Finally, RNA was extracted and purified by the TRIzol method, and the RNA concentration was detected by a NanoDrop 2000 full-spectrum spectrophotometer. Next, the RNAs were reverse transcribed to cDNA and measured by qRT-PCR assay.

2.12. Chromatin Isolation by RNA Purification (ChIRP) Assay. To explore the correlations between lncRNAs and proteins, we performed a ChIRP assay. According to the gene sequence of SEMA3B-AS1, a biotin-labeled ChIRP probe was designed by us and synthesized by RiboBio Co. Ltd. (Guangzhou, China). Then, we performed a ChIRP assay according to a reference book and the protocol of the ChIRP assay kit (Guangzhou, China). The biotin probe sequences of SEMA3B-AS1 are listed in Supplementary Table 1. The protein product of the ChIRP experiment was detected by Western blotting.

2.13. Dual-Luciferase Reporter Assay. We first cloned the FBXW7 promoter into the PGL4.17 basic luciferase reporter vector (Promega, USA), and SEMA3B-AS1 and HMGB1 were cloned into the pCDNA3.1 plasmid. In brief, HEK293 T cells $\left(1 \times 10^{4}\right)$ were seeded into 96-well plates and cultured for $24 \mathrm{~h}$. Then, related plasmids were cotransfected according to the protocol and experimental groups. Each well was transfected with $5 \mathrm{ng}$ Renilla reporter plasmid, $100 \mathrm{ng}$ expression vector (PGL4.27-HMGB1 or PGL3BASIC), and FBXW7 promoter plasmid, which were normalized after cotransfection. After $24 \mathrm{~h}$ of incubation, the luciferase activity of PGL4.17-FBXW7-Pro was detected using a Dual-Luciferase Reporter Gene Analysis System (Promega, USA).

2.14. Coimmunoprecipitation (co-IP) Assay. According to the protocol of the co-IP kit (Abison, Guangzhou, China), the cells needed for the experiment were cultured and collected. After removing the DMEM, the cells were washed once with $1 \mathrm{x}$ PBS. Then, $0.5 \mathrm{~mL}$ of ice-cold lysis buffer was added to the cell plate $(10 \mathrm{~cm})$ and incubated on ice for at least 5 minutes. Next, we scraped off the cells and transferred them to a new EP tube. Then, the follow-up experiment was completed according to the operation process of the kit. Finally, an immunoprecipitation- (IP-) immunoblotting (IB) test was carried out to detect and analyze the binding of the FBXW7 and BGN proteins.

2.15. Animal Experiment. Six- to eight-week-old female nude mice $(B A L B / c)$ were purchased from Jiangsu Jicui Yaokang Biotechnology Co. Ltd. (Nanjing, China). To establish a subcutaneous tumorigenesis model in nude mice, we randomly divided the mice into three groups (OeNC-SEMA3B-AS1HGC, Oe-SEMA3B-AS1-HGC, and Oe-SEMA3B-AS1HGC+SH-FBXW7) ( $n=5$ mice/group). A total of $5 \times 10^{5}$ GC cells (HGC-27) were suspended in $0.1 \mathrm{~mL}$ DMEM and injected into the flank of each mouse. After two weeks, we began to observe subcutaneous tumor formation and assess tumor volume once a week, tumor volume $=1 / 2 \times\left(\right.$ width $^{2}$ $x$ length). At the 5 th week or when the tumor diameter was over $1.5 \mathrm{~cm}$, the mice were euthanized, and the tumors were removed for subsequent experiments. For the intraabdominal tumor model, we randomly divided the mice into three groups (OeNC-SEMA3B-AS1-HGC, Oe-SEMA3BAS1-HGC, and Oe-SEMA3B-AS1-HGC+SH-FBXW7) ( $n=6$ mice/group). A total of $1 \times 10^{6}$ GC cells (HGC-27) were suspended in $0.15 \mathrm{~mL}$ DMEM, and then, the right lower abdominal cavity was selected for injection. Five weeks later, the mice were euthanized. Then, we dissected the mice and evaluated abdominal tumor formation and tumor load. Finally, we assessed the expression of the involved genes in tumor tissues by immunohistochemical staining.

2.16. Statistical Analysis. We used SPSS software (version 23.0, IBM SPSS, USA) and GraphPad Prism (version 8.0, GraphPad Software, USA) to analyze all the data in this study. To evaluate the relationship between SEMA3B-AS1 expression and BGN expression, Pearson's correlation analysis was performed. Then, we utilized the chi-square test to analyze the expression of SEMA3B-AS1 and BGN and the clinicopathological status of GC patients. Two groups were 


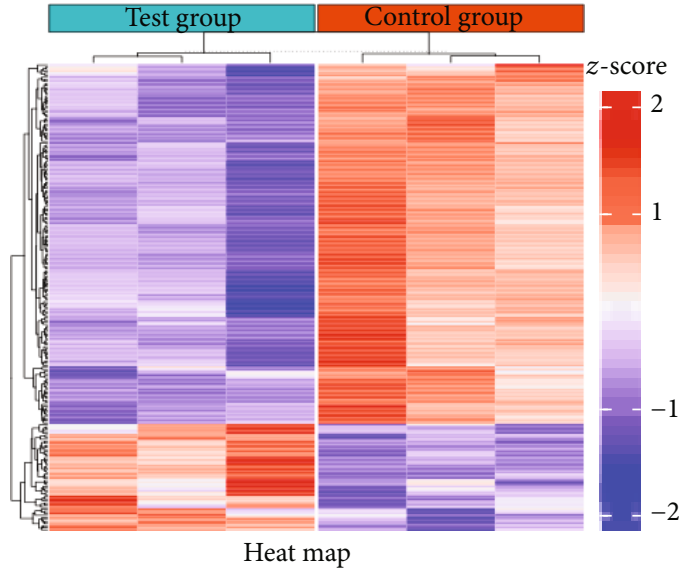

(a)

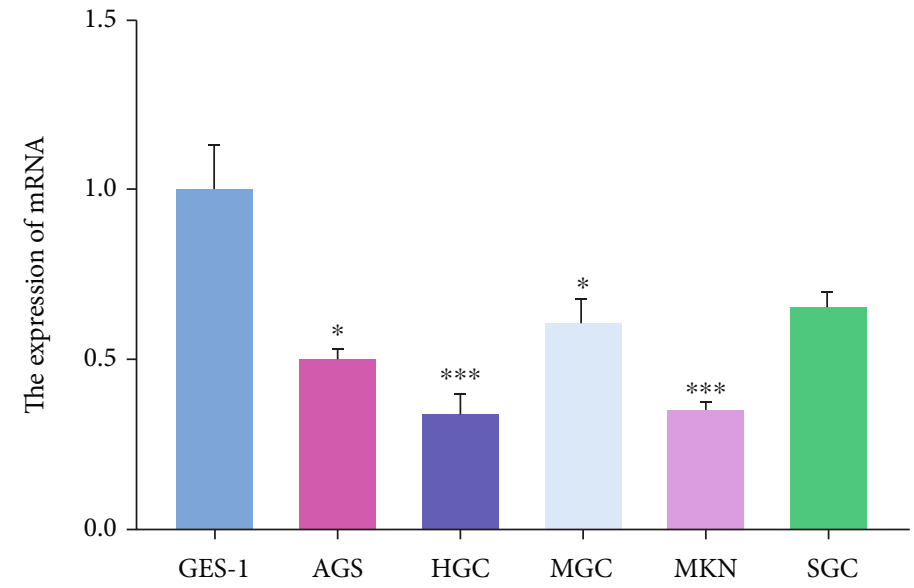

(b)

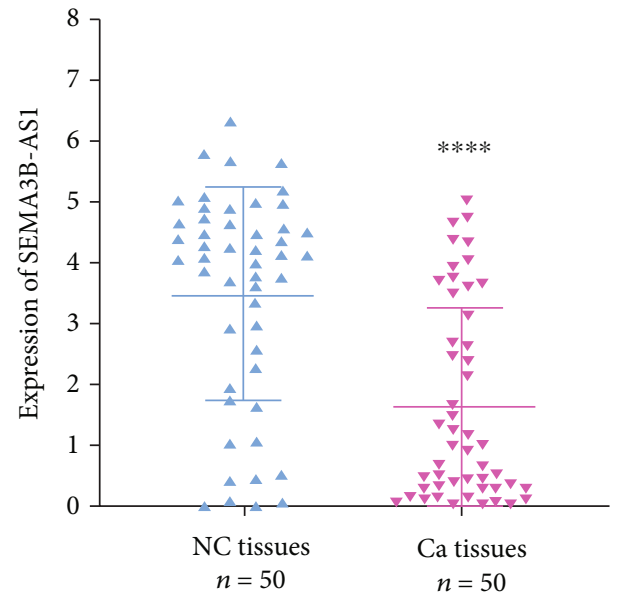

(c)

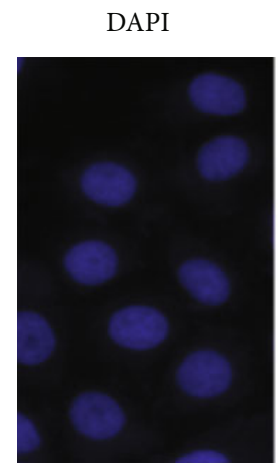

SEMA3B-AS1

Merged
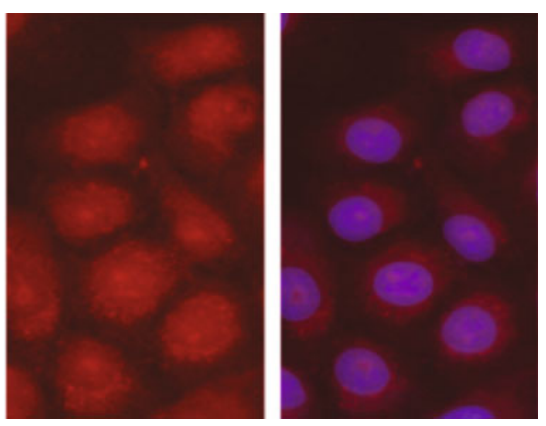

DAPI

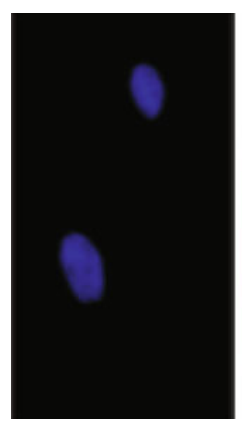

SEMA3B-AS1

Merged

SGC-7901
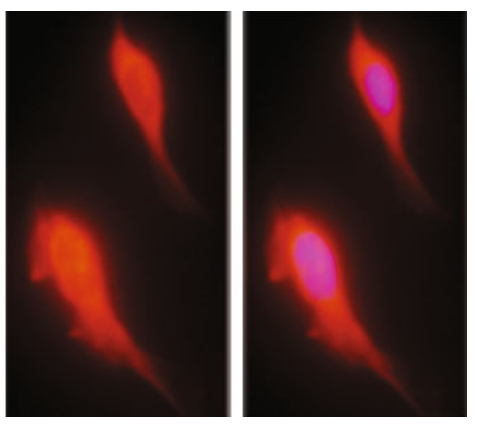

HGC-27

(d)

FIgURE 1: Continued. 

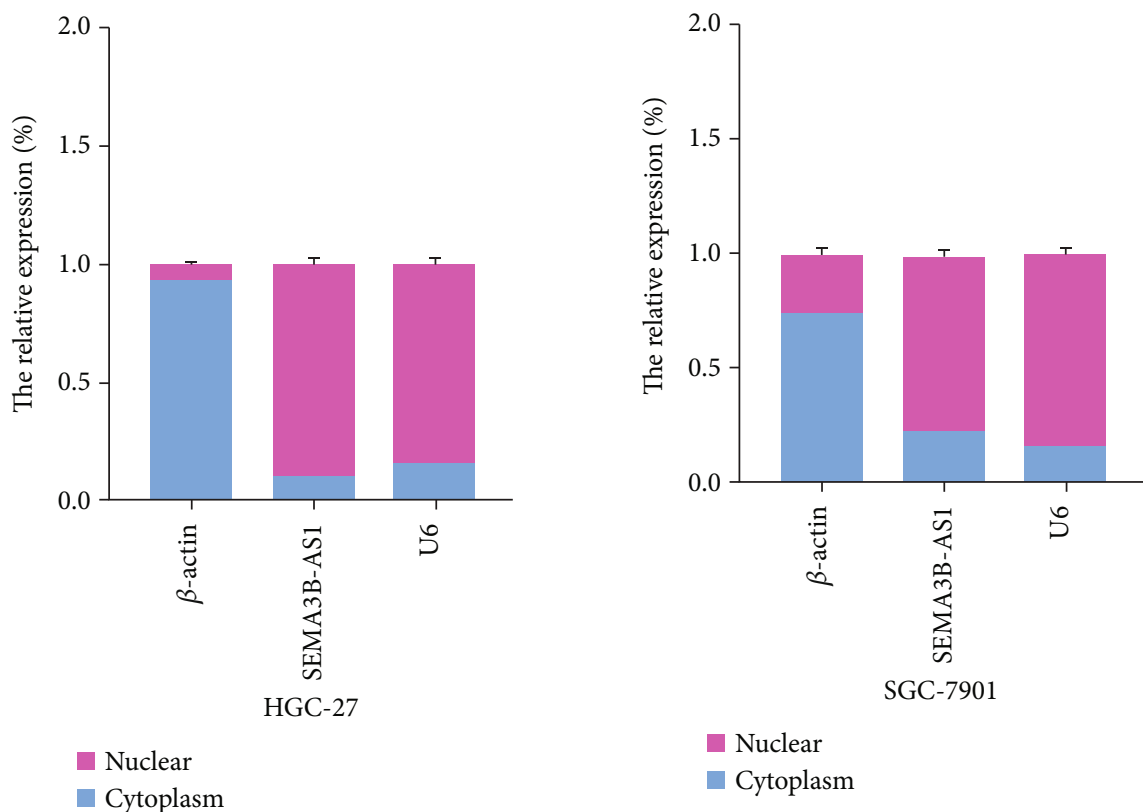

(e)
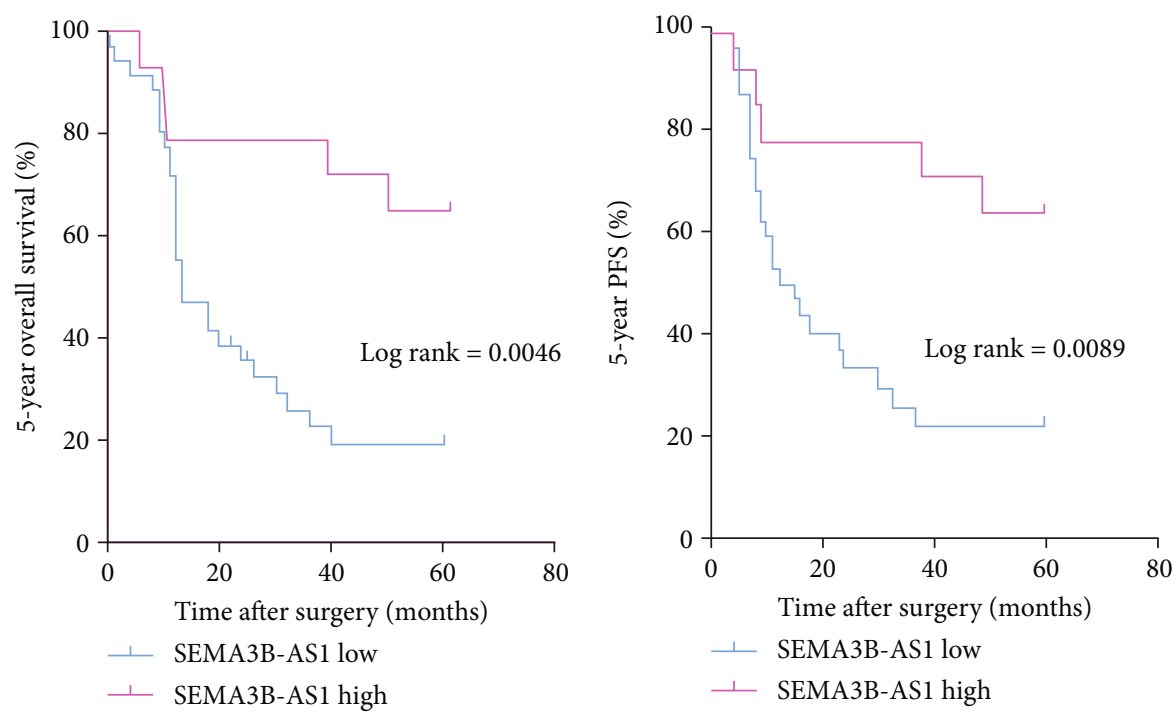

(f)

Figure 1: Continued. 

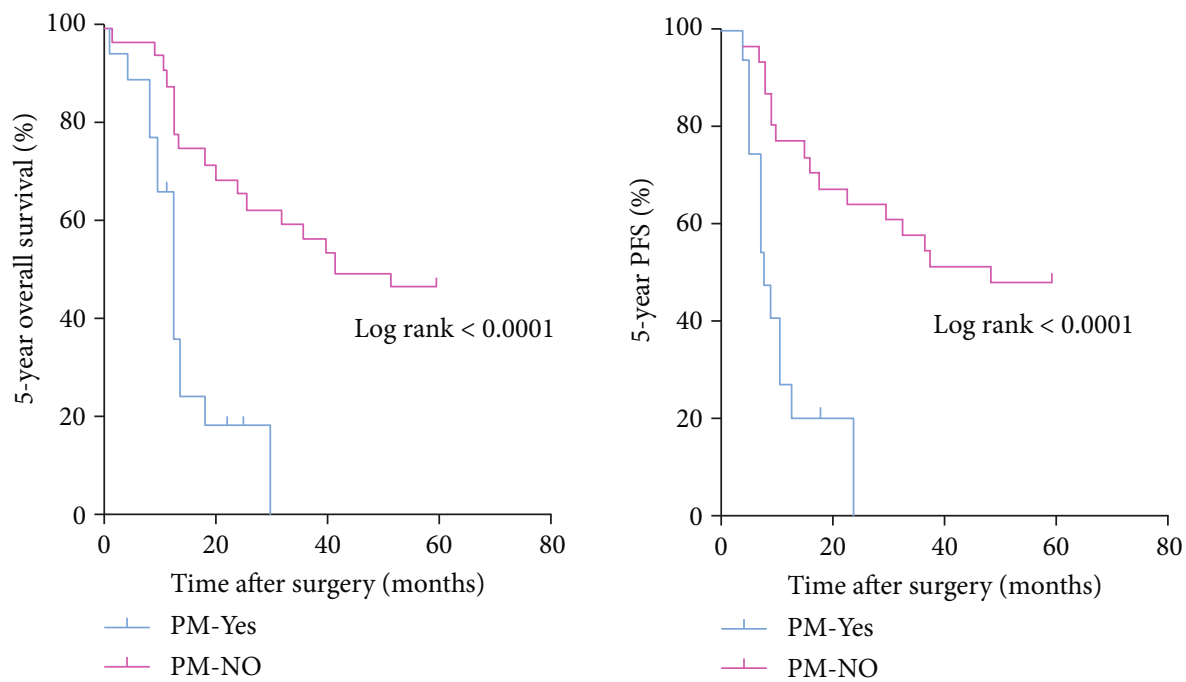

(g)

FIgURE 1: SEMA3B-AS1 was downregulated in human GC tissues. It was mainly located in the nucleus. Data are shown as mean \pm SEM $(n=3) ;{ }^{*} p<0.05,{ }^{* *} p<0.01,{ }^{* * *} p<0.001$, and ${ }^{* * *} p<0.0001$. (a) IncRNA expression was identified in three pairs of GC tissues and corresponding PM of GC tissues by microarray analysis according to the value of the samples and fluorescence intensity. (b) The relative mRNA expression of SEMA3B-AS1 in five GC cell lines and normal stomach epithelial cell line (GES-1) was identified by qRT-PCR assay. (c) The relative mRNA expression of SEMA3B-AS1 was identified by qRT-PCR assay in 50 pairs GC tissues and adjacent normal tissues. (d) The intracellular location of SEMA3B-AS1 was identified by RNA FISH assay (magnification: $\times 400$ ). (e) The nuclear and cytoplasmic distribution of SEMA3B-AS1 in HGC-27 and SGC-7901 cell lines was identified using qRT-PCR assay. (f) The correlation with the expression level of SEMA3B-AS1 between five-year overall survival (OS) and five-year progression-free survival (PFS) was analyzed through Kaplan-Meier survival analysis. (g) The correlation with (PM) between five-year overall survival (OS) and five-year progression-free survival (PFS) was analyzed through Kaplan-Meier survival analysis.

compared using Student's $t$-test, Wilcoxon test, or Fisher's exact test. Comparisons between groups were performed by variance analysis or the chi-square test. Moreover, the Kaplan-Meier survival curve and log-rank test were applied for survival analysis. Finally, the independent factors affecting the prognosis of gastric cancer were determined by using univariate and multivariate Cox regression analyses. All cell experiments were carried out independently at least three times. In all cases, the results were considered statistically significant when the $p$ value $<0.05$.

\section{Results}

3.1. SEMA3B-AS1 Is Downregulated in Human GC Tissues and Mainly Located in the Nucleus. According to the lncRNA sequencing results of our research group, we found that SEMA3B-AS1 expression was decreased in PM tissues compared to primary lesion tissues (Figure 1(a)). Next, we verified the SEMA3B-AS1 mRNA expression level in five gastric cancer cell lines (AGS, HGC-27, MGC-803, MKN45, and SGC-7901) and a normal stomach mucosal cell line (GES-1) through qRT-PCR assay, and it was also demonstrated that SEMA3B-AS1 expression was decreased in GC cells compared to GES-1 cells (Figure 1(b)). Moreover, we detected the mRNA expression level of SEMA3B-AS1 in 50 pairs of GC tissues and corresponding adjacent normal tissues using qRT-PCR assays. The relative mRNA expression of SEMA3B-AS1 was significantly decreased in GC tissues compared with normal tissues, and the difference was statis- tically significant $(p<0.0001)$ (Figure $1(c))$. The function of lncRNAs is closely related to their localization. To determine the localization of SEMA3B-AS1, FISH assays and subcellular fractionation assays (for localization assessment) were carried out in SGC-7901 or HGC-27 cells, and we found that SEMA3B-AS1 was mainly localized in the nucleus (Figures 1(d) and 1(e)). These data demonstrated that SEMA3B-AS1 was mainly localized in the nucleus and downregulated in human GC.

3.2. SEMA3B-AS1 Is Associated with Metastasis and Prognosis in GC Patients. Based on the clinical data of 50 GC patients, we explored the correlations between clinicopathological features and SEMA3B-AS1 expression levels (Table 1). The results showed that the low expression of SEMA3B-AS1 was obviously related to tumor size, $\mathrm{T}$ stage, lymphatic metastasis, distant metastasis, tumor-nodemetastasis (TNM) stage, and PM $(p<0.05)$. However, it was not associated with sex, age, cancer grade, CEA, or CA19-9 ( $p>0.05)$. Moreover, the survival analysis demonstrated that patients with low SEMA3B-AS1 expression had much shorter 5-year overall survival (OS) (Figure 1(f)) and 5-year progression-free survival (PFS) (Figure 1(f)) times than those who had high SEMA3B-AS1 expression. The 5-year overall survival rate analysis showed that the survival rate of patients with low SEMA3B-AS1 expression in cancer tissues was significantly lower than that of patients with high SEMA3B-AS1 expression in cancer tissues $(9 / 27$ $(33.33 \%)$ vs. $9 / 14(64.29 \%) \quad p<0.05)$. Univariate and 
TABLE 1: The correlation of SEMA3B-AS1 expression with the clinicopathological parameters of GC patients $(n=50)$.

\begin{tabular}{|c|c|c|c|c|}
\hline \multirow[t]{2}{*}{ Parameters } & \multirow[t]{2}{*}{$n(\%)$} & \multicolumn{2}{|c|}{$\begin{array}{c}\text { SEMA3B-AS1 } \\
\text { expression }\end{array}$} & \multirow[t]{2}{*}{$p$ value } \\
\hline & & Low & High & \\
\hline \multicolumn{5}{|l|}{ Gender } \\
\hline Male & $34(68)$ & 25 & 9 & \multirow{2}{*}{0.726} \\
\hline Female & $16(32)$ & 11 & 5 & \\
\hline \multicolumn{5}{|l|}{ Age } \\
\hline$\geq 60$ years & $34(68)$ & 24 & 10 & \multirow[t]{2}{*}{0.746} \\
\hline$<60$ years & $16(32)$ & 12 & 4 & \\
\hline \multicolumn{5}{|l|}{ Cancer grade } \\
\hline Moderate/well & $10(20)$ & 6 & 4 & \multirow{2}{*}{0.345} \\
\hline Poor & $40(80)$ & 30 & 10 & \\
\hline \multicolumn{5}{|c|}{ Cancer size $(\mathrm{cm})$} \\
\hline$\geq 5$ & $13(26.0)$ & 13 & 0 & \multirow{2}{*}{0.009} \\
\hline$<5$ & $37(74.0)$ & 23 & 14 & \\
\hline \multicolumn{5}{|l|}{$\mathrm{T}$ stage } \\
\hline T1-2 & $11(22.0)$ & 4 & 7 & \multirow{2}{*}{0.003} \\
\hline T3-4 & $39(78.0)$ & 32 & 7 & \\
\hline \multicolumn{5}{|c|}{ Lymphatic metastasis } \\
\hline N0-1 & $23(46.0)$ & 13 & 10 & \multirow{2}{*}{0.024} \\
\hline $\mathrm{N} 2-3$ & $27(54.0)$ & 23 & 4 & \\
\hline \multicolumn{5}{|c|}{ Distant metastasis } \\
\hline Yes & $11(22.0)$ & 11 & 0 & \multirow{2}{*}{0.019} \\
\hline No & $39(88.0)$ & 25 & 14 & \\
\hline \multicolumn{5}{|l|}{ TNM stage } \\
\hline $\mathrm{I} / \mathrm{II}$ & $17(34.0)$ & 7 & 10 & \multirow{2}{*}{$<0.001$} \\
\hline III/IV & $33(66.0)$ & 29 & 4 & \\
\hline \multicolumn{5}{|l|}{ CEA (ng/mL) } \\
\hline$\geq 5$ & $9(18.0)$ & 6 & 3 & \multirow{2}{*}{0.694} \\
\hline$<5$ & $41(82.0)$ & 30 & 11 & \\
\hline \multicolumn{5}{|l|}{ CA19-9 (u/mL) } \\
\hline$<37$ & $40(80.0)$ & 28 & 12 & \multirow{2}{*}{0.529} \\
\hline$\geq 37$ & $10(20.0)$ & 8 & 2 & \\
\hline \multicolumn{5}{|l|}{ PM } \\
\hline Yes & $19(38.0)$ & 18 & 1 & \multirow{2}{*}{0.005} \\
\hline No & $31(62.0)$ & 18 & 13 & \\
\hline
\end{tabular}

Remark: tumor staging guidelines: AJCC Cancer Staging Manual (8th). Abbreviations: TNM: tumor-node-metastasis; CEA: carcinoembryonic antigen; CA19-9: carbohydrate antigen 19-9; $\mathrm{T}$ stage: tumor invasion stage; PM: peritoneal metastasis. $p<0.05$ : statistically significant.

multivariate analyses revealed that SEMA3B-AS1 expression was obviously related to poor PFS (HR: 0.123, 95\% CI: $0.023-0.662, p=0.015)$ and OS (HR: $0.113,95 \%$ CI: $0.021-$ $0.603, p=0.011$ ) (Table 2). These results demonstrated that SEMA3B-AS1 is significantly correlated with the progression and metastasis of GC.

3.3. SEMA3B-AS1 Inhibits the Activity, Invasion, Proliferation, and Migration and EMT of GC Cells In Vitro. Given the important role of SEMA3B-AS1 in metastasis, OS, and PFS of GC patients, gain- or loss-of-function tests were performed to verify the biological functions of SEMA3B-AS1. According to the expression characteristics of SEMA3B-AS1 in GC cell lines, SGC-7901 was selected as the knockdown model cell line, and HGC-27 was selected as the overexpression model cell line (Figure 1(b)). Then, stable cell lines were constructed and named LV-SHSEMA3B-AS1-SGC and Lv-Oe-SEMA3B-AS1-HGC. Next, we detected the efficiency of overexpression and knockdown of SEMA3B-AS1 through qRT-PCR assay (Figures 2(a) and 2(b)).

The viability, proliferation, invasion, and migration of GC cells were detected by CCK-8 assay, colony formation assay, transwell assay, and wound healing assay, respectively, after SEMA3B-AS1 overexpression. The results showed that the viability (Figure 2(c)), proliferation (Figure 2(d)), migration (Figure 2(e)), and invasion (Figure 2(f)) of SEMA3BAS1-overexpressing cells were obviously decreased compared with those of the negative control group. In contrast, after knockdown of SEMA3B-AS1 in SGC cells, the viability (Figure 2(g)), proliferation (Figure 2(h)), migration (Figure 2(i)), and invasion (Figure 2(j)) abilities of GC cells were increased. Previous experimental results showed that SEMA3B-AS1 was significantly associated with lymph node metastasis and PM in patients with GC. We speculated that SEMA3B-AS1 might be closely related to the EMT ability of tumor cells. Thus, we detected EMT indicators, including vimentin and E-cadherin, in HGC-27 or SGC-7901 cells after SEMA3B-AS1 overexpression or knockdown using Western blotting. The results demonstrated that the protein expression of E-cadherin was increased, while that of vimentin was decreased after SEMA3B-AS1 overexpression (Figure 2(k)). However, in SEMA3B-AS1 knockdown cells, we obtained the exact opposite results (Figure 2(l)). These data demonstrated that the overexpression of SEMA3BAS1 could decrease the viability, invasion, proliferation, migration, and EMT of GC cells.

3.4. SEMA3B-AS1 Can Combine with HMGB1. Based on a previous study, we demonstrated that SEMA3B-AS1 plays a vital role in GC progression and metastasis, but the exact mechanism is unclear. Existing research has demonstrated that lncRNAs bind to DNA, RNA, and proteins to regulate gene expression via multiple mechanisms, including epigenetic regulation, transcriptional regulation, and posttranscriptional regulation [27]. To explore the mechanism of SEMA3B-AS1, we conducted a biotin RNA pull-down experiment to identify potential proteins binding to SEMA3B-AS1 (Figure 3(a)). By MS analysis, we found that HMGB1, a multifunctional molecule with different roles at different sites, was an interacting protein of SEMA3B-AS1 (Supplementary Table 2, RNA pull-down-MS). To further confirm the mutual binding of SEMA3B-AS1 and HMGB1, we conducted RIP experiment. We found significant enrichment of HMGB1 with the anti-HMGB1 antibody versus IgG in HGC-27 or SGC-7901 GC cells (Figures 3(b) and 3(c)). Moreover, we demonstrated the interaction between SEMA3B-AS1 and HMGB1 using a ChIRP assay. We found significant enrichment of HMGB1 in the probe group targeting SEMA3B-AS1 relative to the negative 


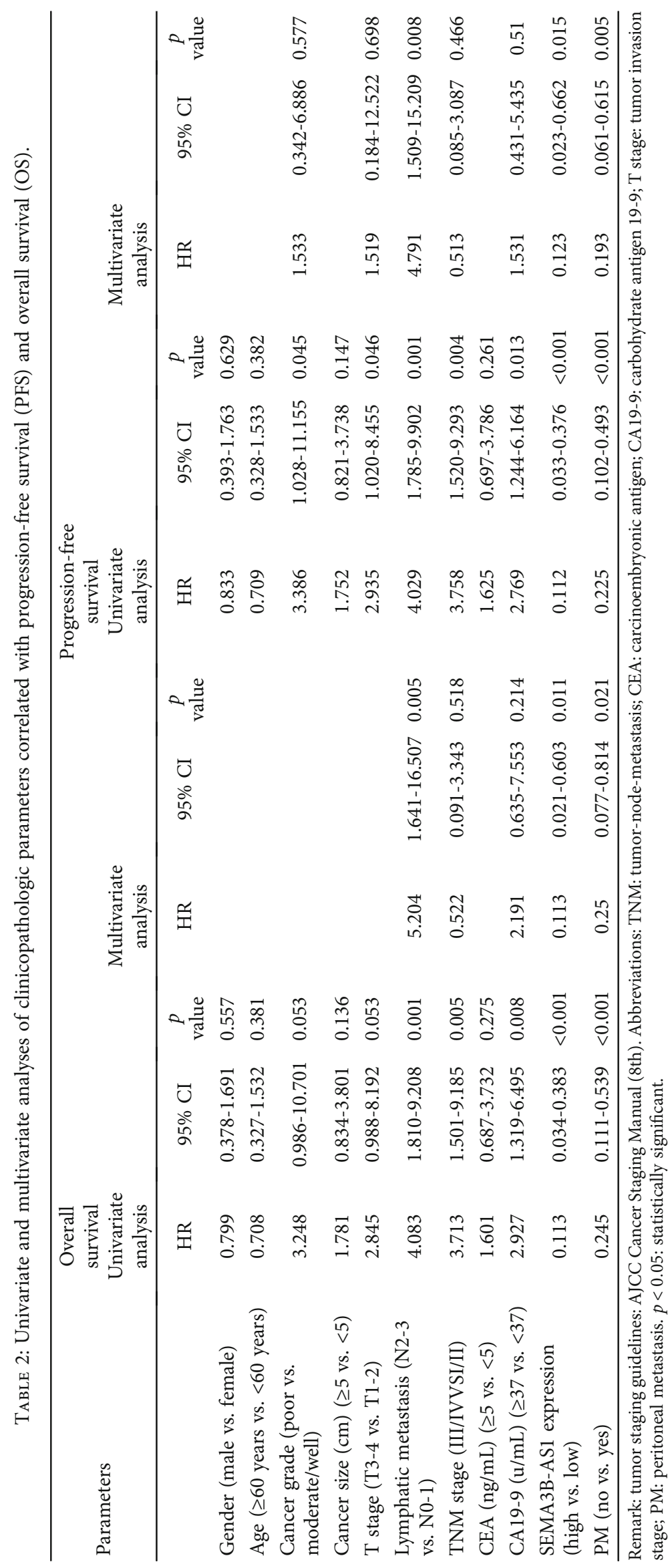




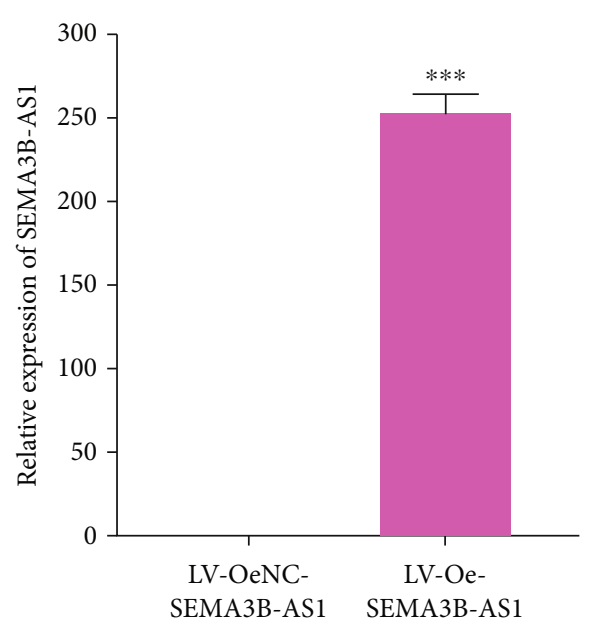

HGC-27

(a)

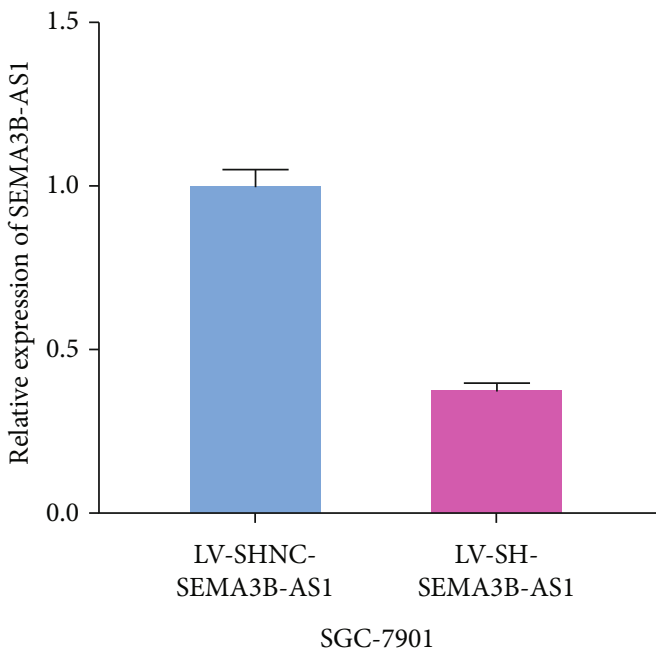

(b)

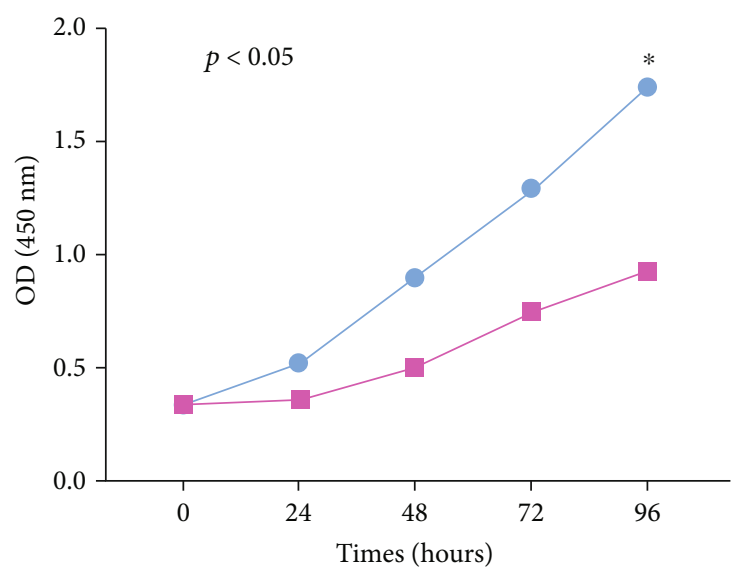

- OeNc-SEMA3B-AS1-HGC27

$\rightarrow$ Oe-SEMA3B-AS1-HGC27

(c)
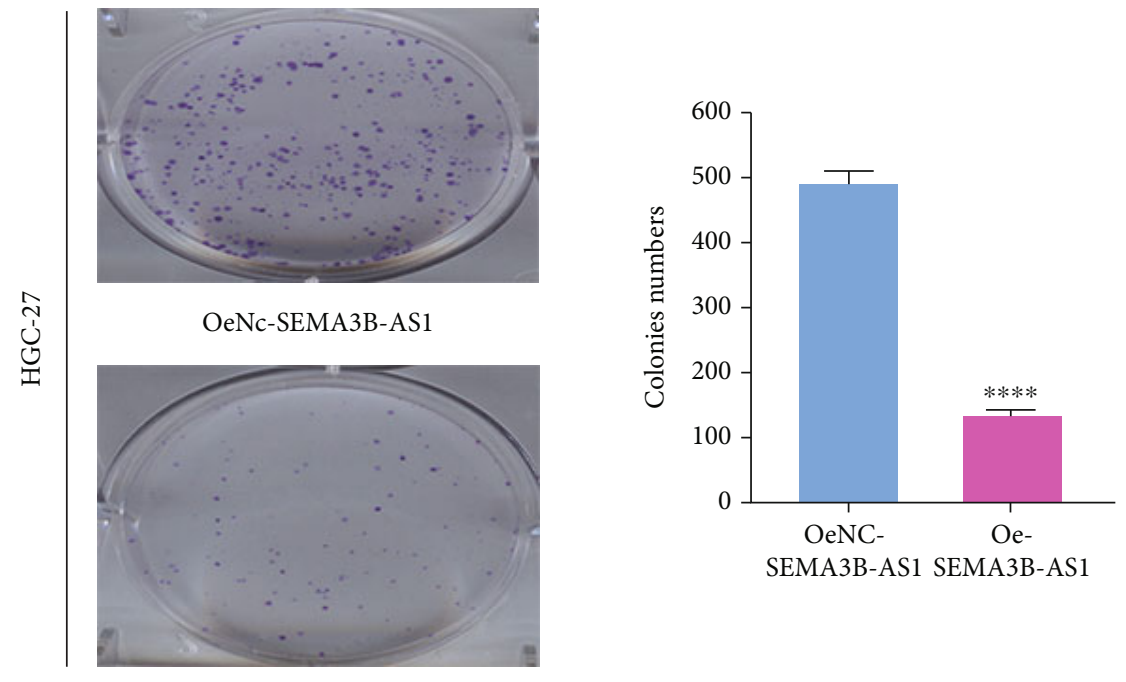

Oe-SEMA3B-AS1

(d)

Figure 2: Continued. 
$0 \mathrm{~h}$

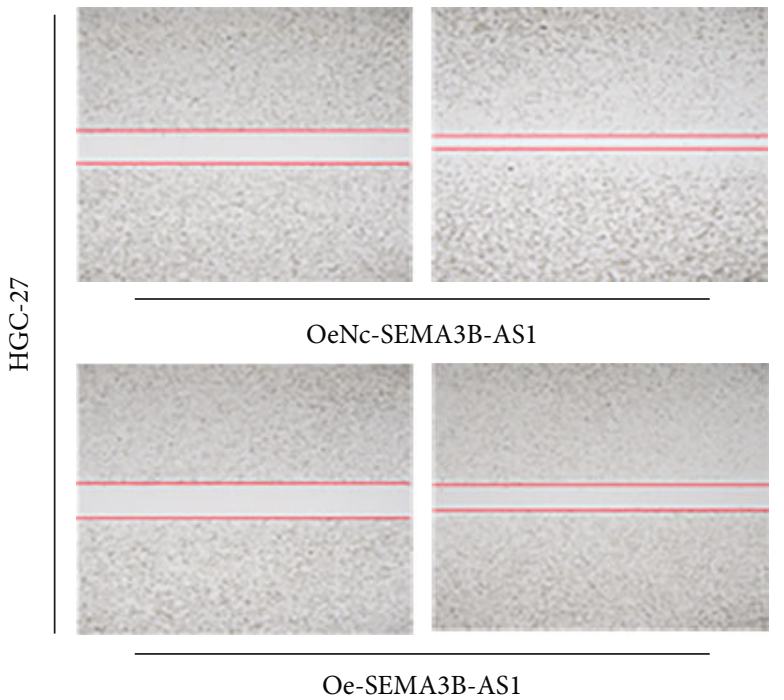

(e)

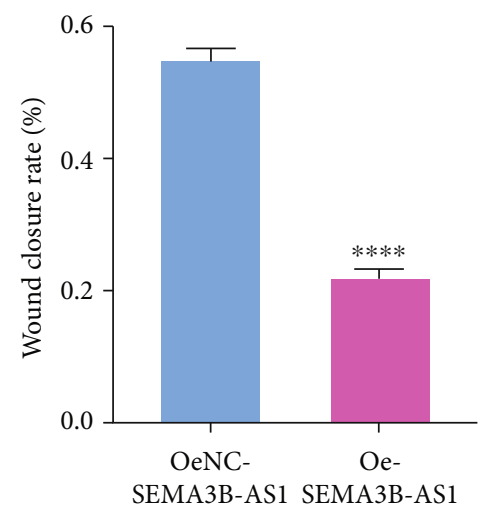

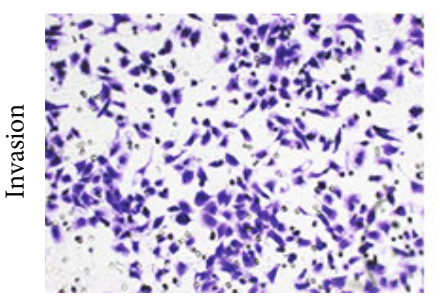

OeNC-SEMA3B-AS1

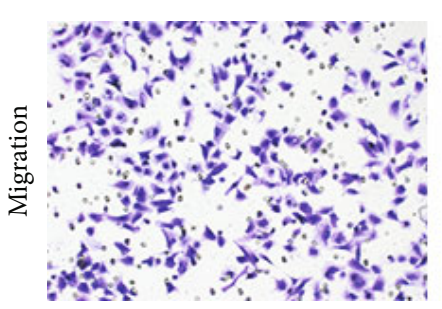

OeNC-SEMA3B-AS1

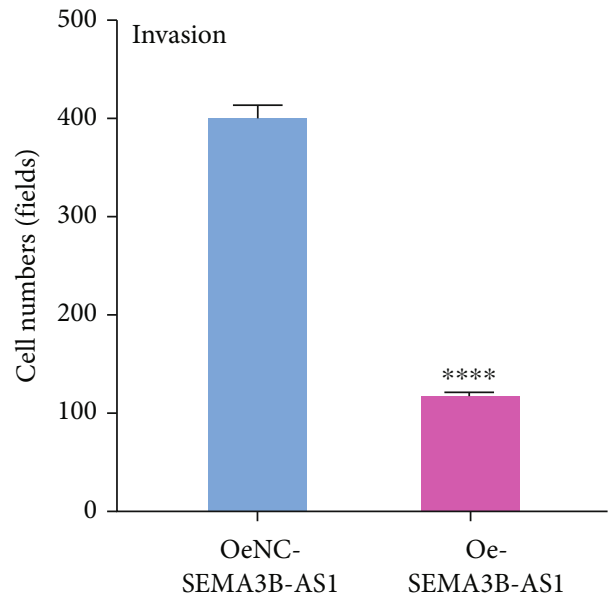

(f)

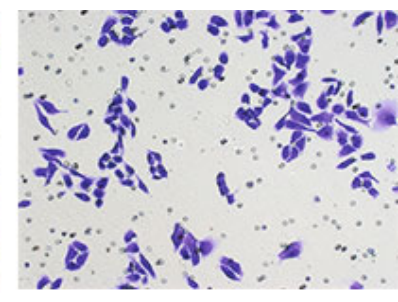

Oe-SEMA3B-AS1

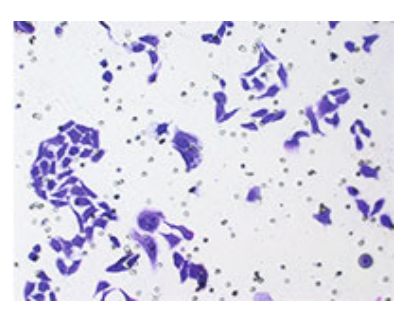

Oe-SEMA3B-AS1

命

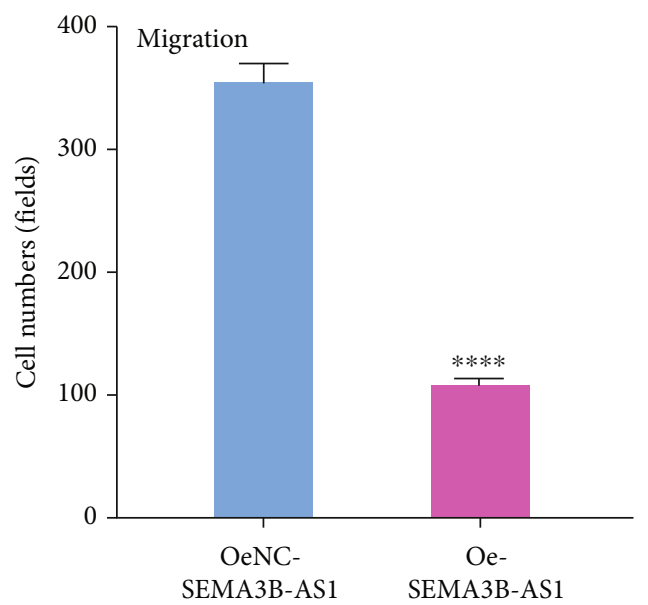

Figure 2: Continued. 


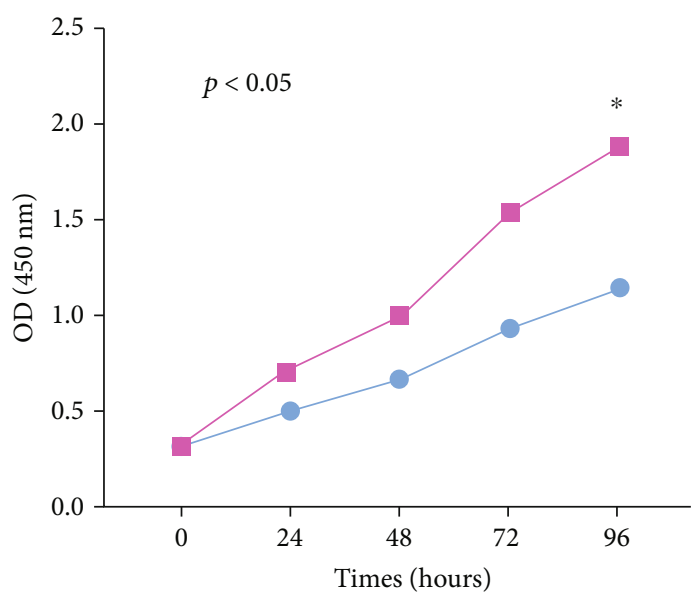

$\longrightarrow$ SHNC-SEMA3B-AS1-SGC-7901

- - SH-SEMA3B-AS1-SGC-7901

(g)

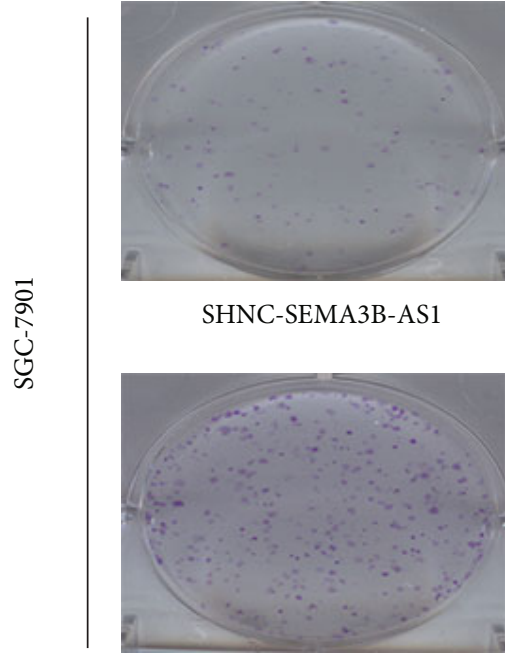

SH-SEMA3B-AS1

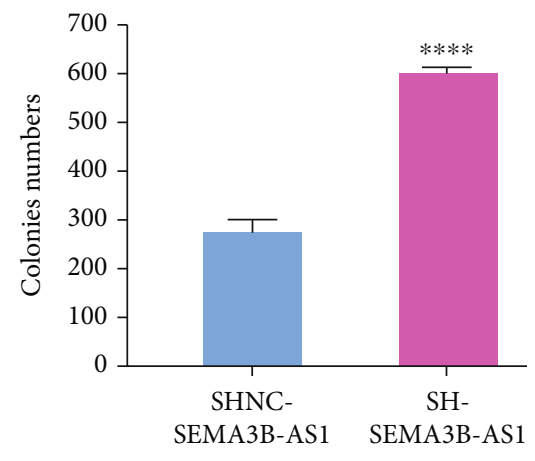

(h)

Figure 2: Continued. 
$0 \mathrm{~h}$

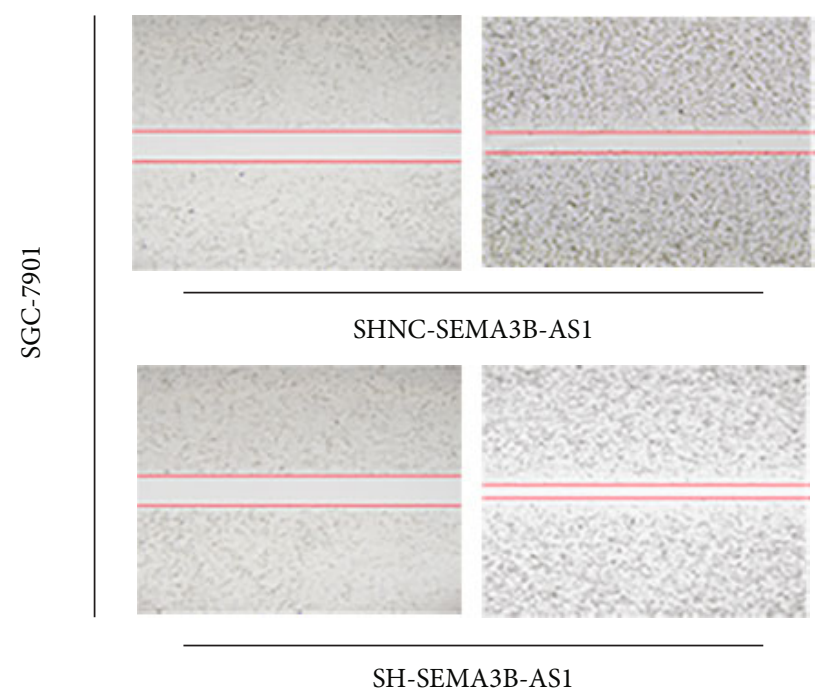

$48 \mathrm{~h}$

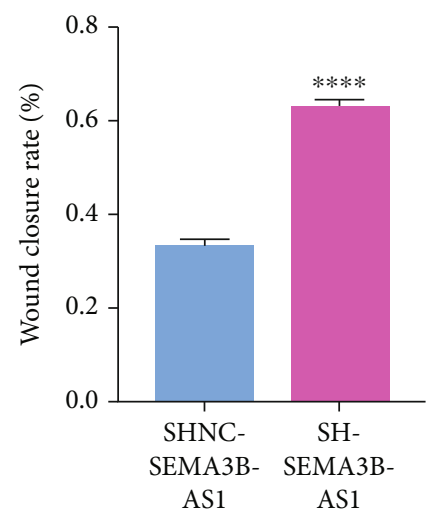

(i)

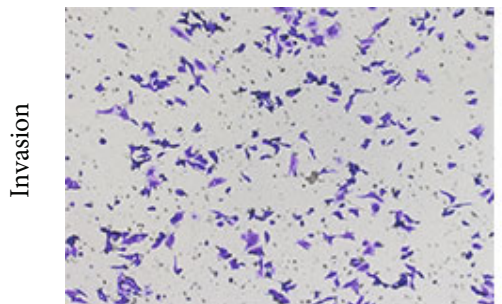

SHNC-SEMA3B-AS1

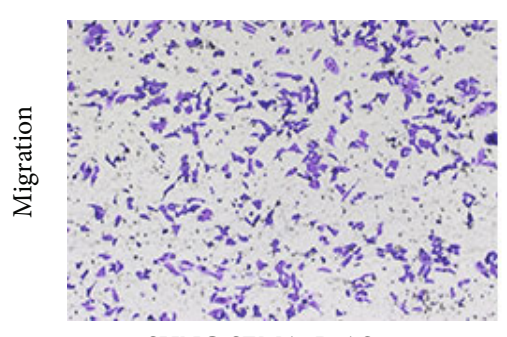

SHNC-SEMA3B-AS1

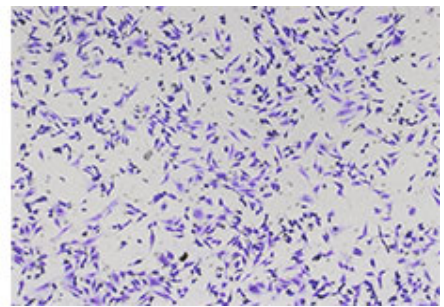

SH-SEMA3B-AS1

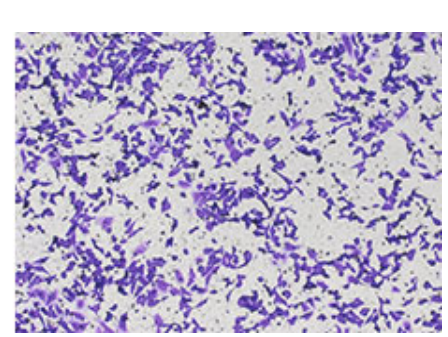

SH-SEMA3B-AS1
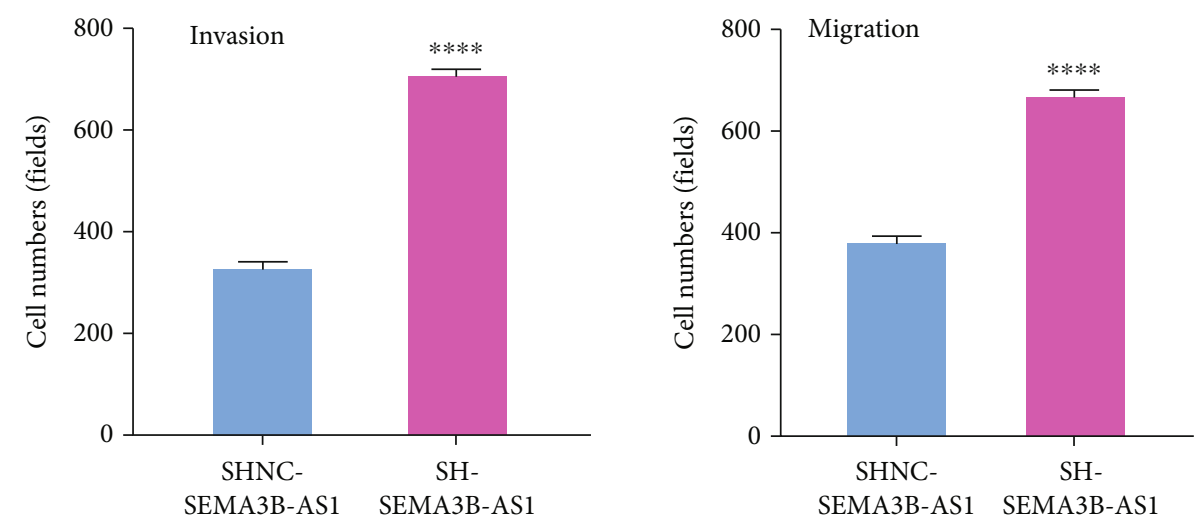

(j)

Figure 2: Continued. 


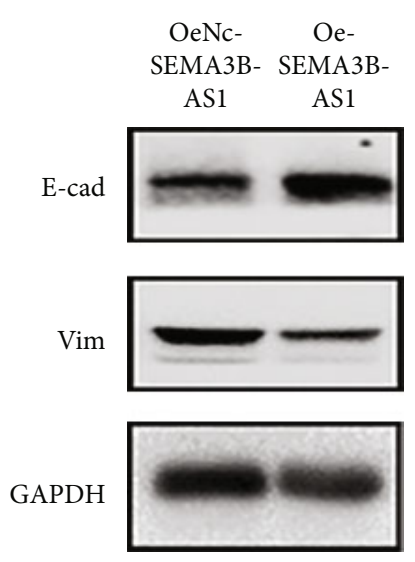

HGC-27

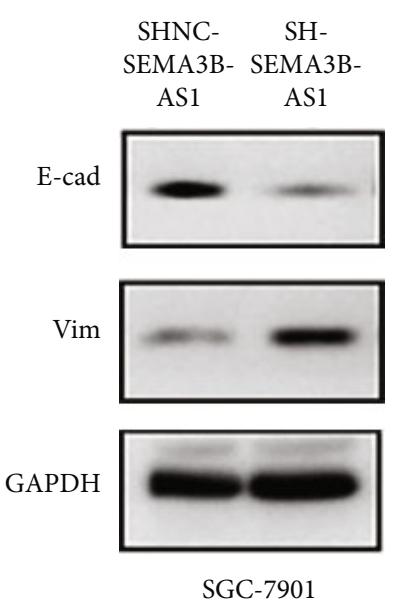

(l)

FIGURE 2: SEMA3B-AS1 could inhibit the activity, invasion, proliferation, and migration and EMT ability of GC cells in vitro (magnification: $\times 200)$. Data were shown as mean $\pm \operatorname{SEM}(n=3) ;{ }^{*} p<0.05,{ }^{* *} p<0.01,{ }^{* * *} p<0.001$, and ${ }^{* * * *} p<0.0001$. (a) The overexpression efficiency of Lv-Oe-SEMA3B-AS1 and negative control (NC) was detected in HGC-27 using qRT-PCR. (b) The expression efficiency of Lv-SH-SEMA3BAS1 and negative control (NC) was detected in SGC-7901 using qRT-PCR. (c) The viability of HGC-27 cells after SEMA3B-AS1 overexpression and negative control (NC) was detected by CCK-8 assay. (d-f) The cell proliferation, migration and invasion ability of GC cells (HGC-27) negative control and SEMA3B-AS1 overexpression were detected by the colony formation assay (d), wound healing assay (e), and transwell assay (f), respectively. (g) The viability of SGC-7901 cells after SEMA3B-AS1 knockdown and negative control (NC) was detected by CCK-8 assay. (h-j) The cell proliferation,migration, and invasion ability of GC cells (SGC-7901) negative control and SEMA3B-AS1 knockdown were detected by the colony formation assay (h), wound healing assay (i), and transwell assay (J), respectively. (k) The expression of EMT markers (E-cadherin and vimentin) in GC cells (HGC-27) was analyzed by Western blotting assay after SEMA3B-AS1 overexpression and negative control. (l) The expression of EMT markers (E-cadherin and vimentin) in GC cells (SGC-7901) was analyzed by Western blotting assay after SEMA3B-AS1 knockdown and negative control.

control probe by testing the protein product of the ChIRP experiment through Western blotting (Figures 3(d) and 3(e)). These data revealed that SEMA3B-AS1 could bind with the HMGB1 protein.

3.5. SEMA3B-AS1 Promotes the Expression of FBXW7 by Binding HMGB1. In our previous study, FBXW7 was found to play a crucial role in the occurrence and development of GC patients, and overexpression of FBXW7 inhibited the progression of GC $[19,28]$. Therefore, we continued to explore whether SEMA3B-AS1 combined with HMGB1 could regulate the expression of FBXW7 to further clarify the regulatory mechanism of FBXW7 in GC. First, we constructed HMGB1 overexpression and interference plasmids and measured the efficiency of interference and overexpression in SGC and HGC cells, respectively (Figures 4(a) and 4(b)). Then, we used Western blotting assay to detect HMGB1 protein changes in cytoplasm and nucleus in the SEMA3B-AS1 overexpressed or interfered GC cells; the results showed that the HMGB1 protein in the nucleus was significantly increased, when SEMA3B-AS1 was overexpressed, while SEMA3B-AS1 was knocked down, the HMGB1 protein in the nucleus was significantly decreased (Figures 4(c) and 4(d)). Next, since the biological function of HMGB1 is related to its localization, we needed to identify the localization status of HMGB1 in gastric cancer cells when SEMA3B-AS1 was overexpressed or knocked down. Localization analysis of HMGB1 by immunofluorescence showed that HMGB1 was mainly located in the nucleus when SEMA3B-AS1 was overexpressed (Figure 4(e)). Yet when SEMA3B-AS1 was knocked down, HMGB1 was displaced mainly in the cytoplasm (Figure 4(f)). To further confirm that HMGB1 can regulate the transcription of FBXW7 gene, we cloned the FBXW7 promoter into the PGL4.17 basic luciferase reporter vector and cloned SEMA3B-AS1 and HMGB1 into the pCDNA3.1 plasmid. Next, the luciferase activity of PGL4.17-FBXW7-Pro was detected using a Dual-Luciferase Reporter Gene Analysis System. The results demonstrated that when SEMA3B-AS1 was combined with HMGB1, the transcription of FBXW7 was significantly enhanced, and the difference between them was statistically significant $(p<0.05)$ (Figure $4(\mathrm{~g}))$. Moreover, the changes in FBXW7 protein were detected by Western blotting assay after overexpression of SEMA3B-AS1 or combined overexpression and interference of HMGB1. The results showed that the protein level of FBXW7 was greatly increased after combined overexpression of SEMA3B-AS1 and HMGB1 (Figure 4(h)). These data demonstrated that SEMA3B-AS1 could facilitate FBXW7 expression by combining with HMGB1.

3.6. SEMA3B-AS1 Might Destabilize the BGN Protein by Regulating FBXW7, Thus Promoting the Ubiquitination Degradation of BGN and Inhibiting GC. We found that BGN protein was significantly overexpressed in PM tissues by MS analysis of primary tumors and GC PM samples. Then, the expression of BGN protein in GC tissues and adjacent normal tissues was analyzed by bioinformatics using GSE54129 data set. The results showed that BGN protein was significantly overexpressed in GC tissues $(p<0.0001)$ 


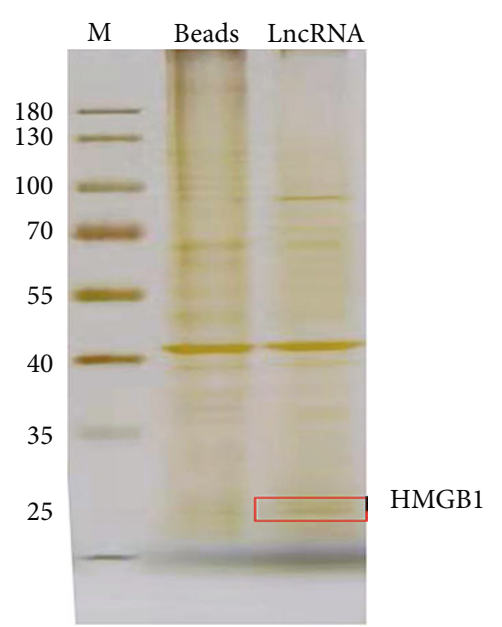

(a)

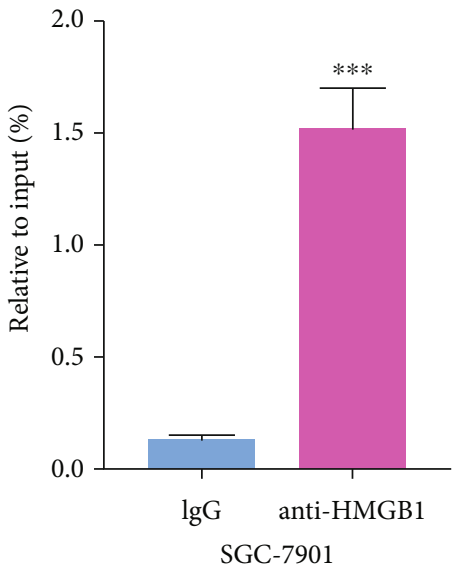

(c)

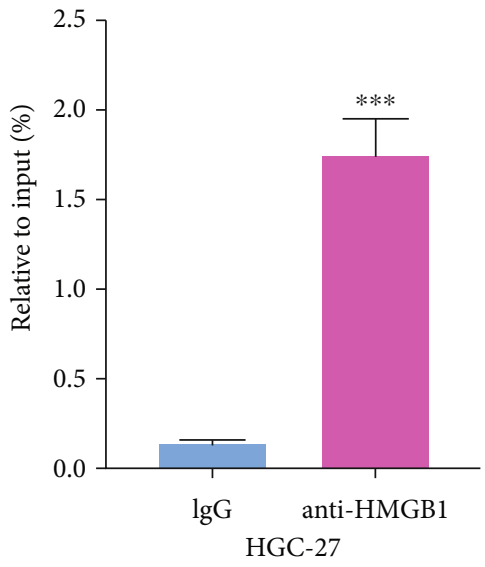

(b)

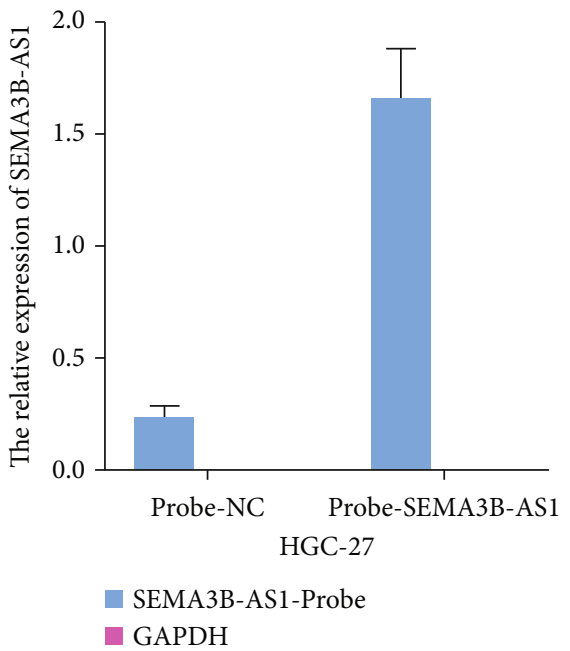

(d)

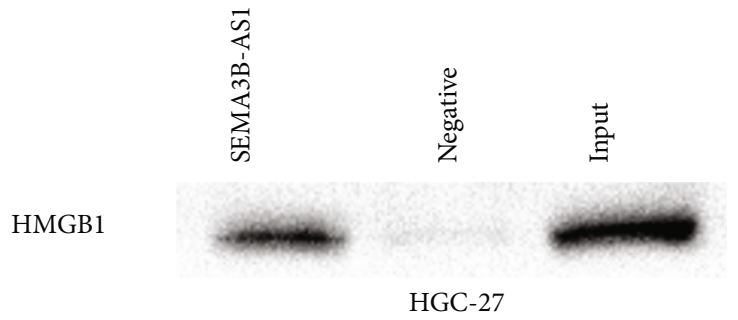

(e)

Figure 3: SEMA3B-AS1 can combine with HMGB1. Data are shown as mean \pm SEM $(n=3) ;{ }^{*} p<0.05,{ }^{* *} p<0.01,{ }^{* * *} p<0.001$, and ${ }^{* * * *} p<0.0001$. (a) RNA pull-down assay showed that biotinylated SEMA3B-AS1 could bind with HMGB1 in GC cells (HGC-27) in vitro. RNA immunoprecipitation (RIP) assay demonstrated the direct binding relationship between SEMA3B-AS1 and HMGB1 in (b) HGC-27 and (c) SGC-7901 cells; the RIP RNA products were detected by qRT-PCR for SEMA3B-AS1. The fold enrichment of SEMA3B-AS1 was relative to its corresponding IgG control. (d) The enrichment of SEMA3B-AS1 in the probe group targeting SEMA3B-AS1 relative to NC probe in HGC-27 cells, as detected by ChIRP assay. GAPDH served as a negative control (NC). (e) The enrichment of HMGB1 protein in the probe group targeting SEMA3B-AS1 relative to the NC probe in HGC-27 cells, as detected by ChIRP assay and the protein product was identified through Western blotting.

(Figure 5(a)). Furthermore, BGN expression was detected in 50 pairs of GC and adjacent normal tissues, and the results also demonstrated that BGN was highly expressed in GC tissues compared to adjacent normal tissues (Figure 5(b)). Then, we used immunohistochemistry to detect BGN expression in normal adjacent tissues, GC tissues, and PM tissues. The results demonstrated that BGN expression was the highest in PM tissues (Figure 5(c)). Existing studies have shown that protein degradation is mainly affected by posttranscriptional modifications, including ubiquitination, 


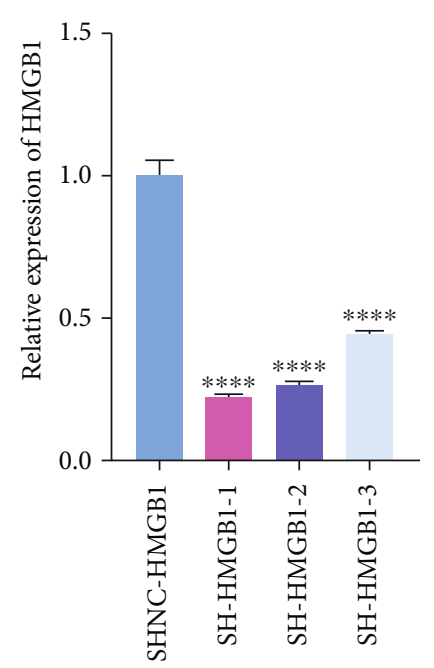

(a)

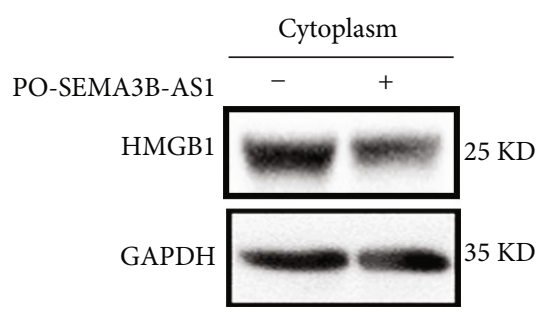

(c)

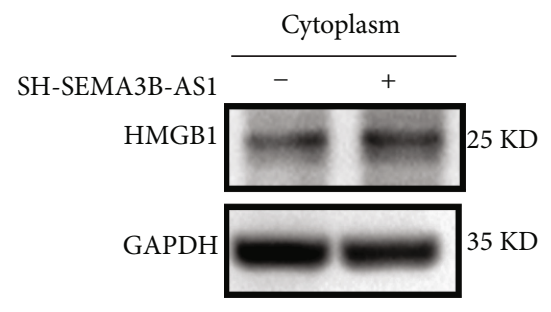

(d)
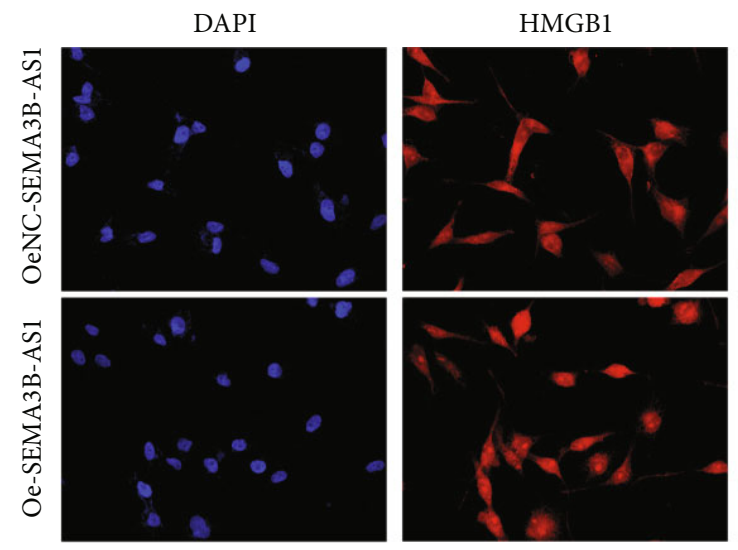

(e)

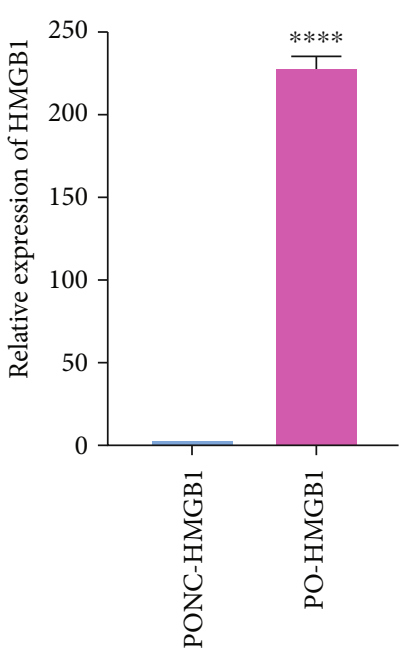

(b)
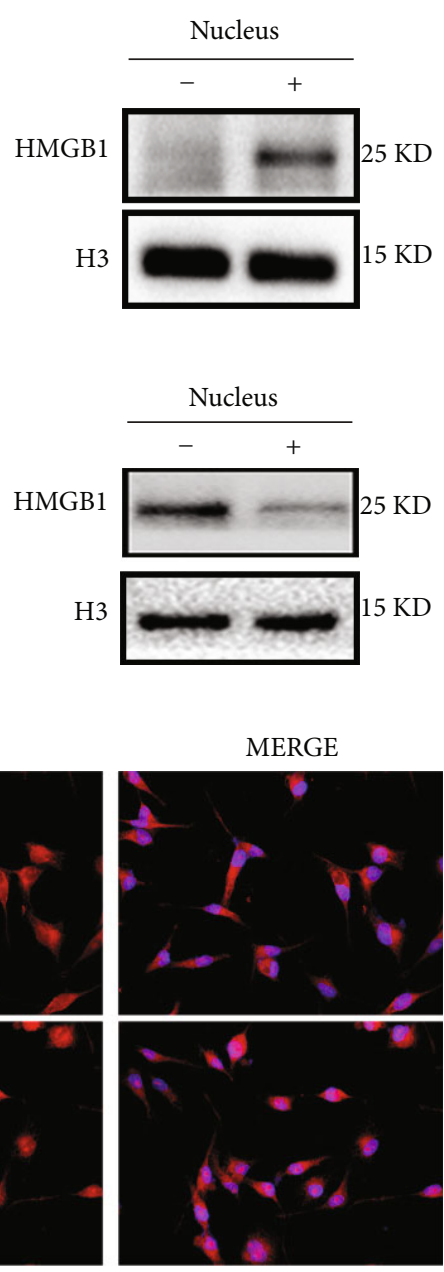

FIgURE 4: Continued. 

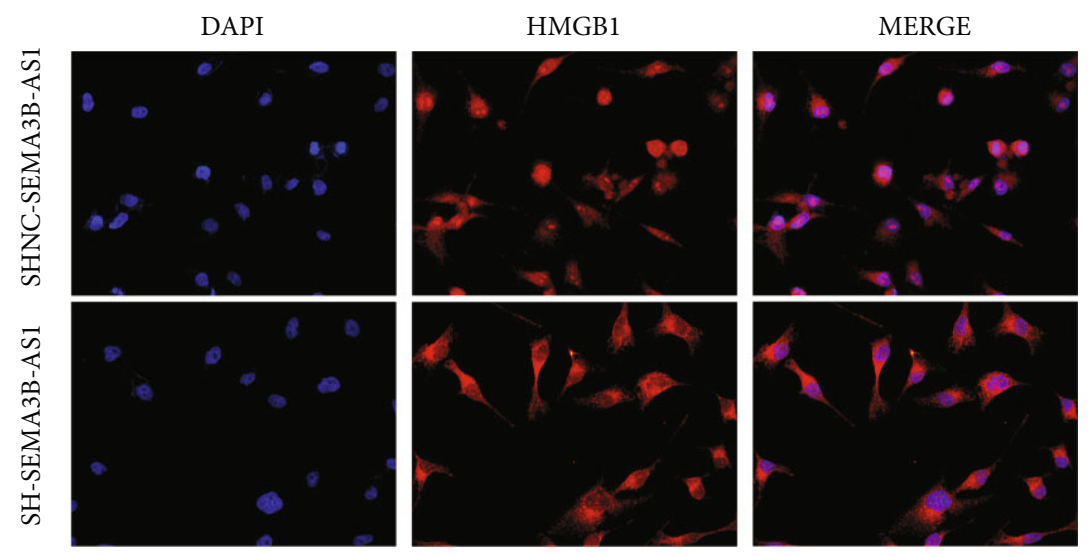

(f)

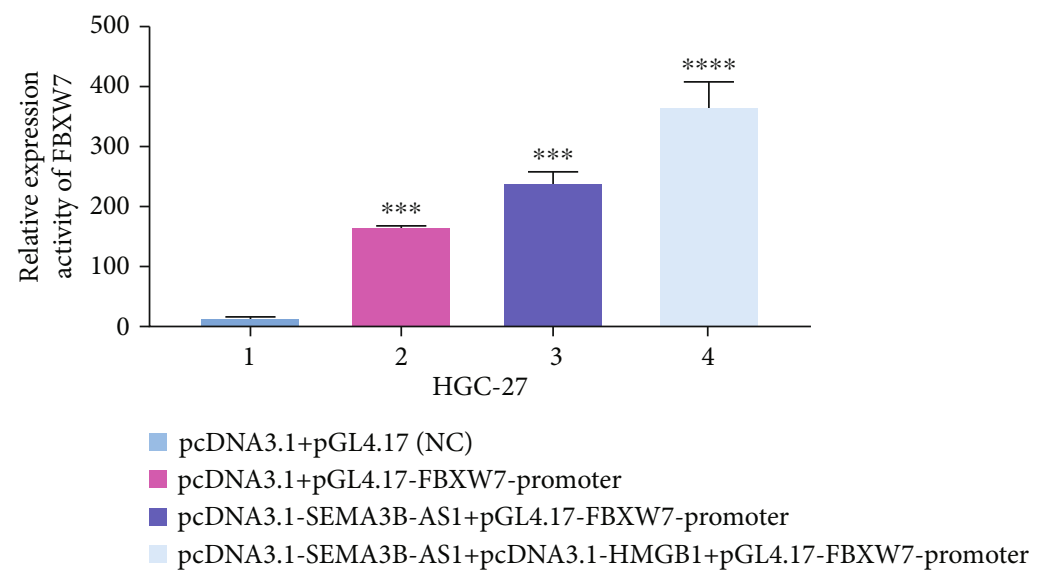

(g)

$\begin{array}{ll}\text { OeNC-SEMA3B-AS1 + } \\ \text { Oe-SEMA3B-AS1 } & - \\ \text { si-HMGB1 } & - \\ \text { Oe-HMGB1 } & -\end{array}$

-
+
+
-

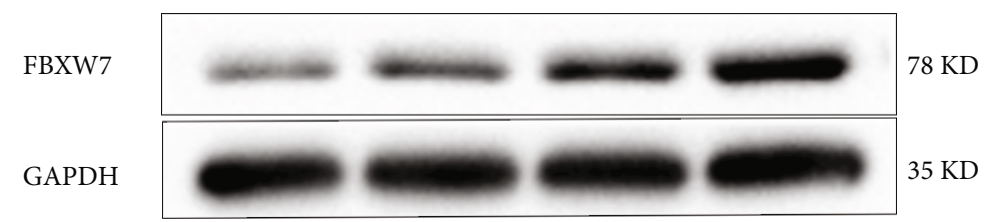

(h)

FiguRE 4: SEMA3B-AS1 promotes the expression of FBXW7 by binding HMGB1. Data are shown as mean \pm SEM $(n=3)$; ${ }^{*} p<0.05,{ }^{* *} p$ $<0.01,{ }^{* * *} p<0.001$, and ${ }^{* * * *} p<0.0001$. (a) The efficiency of HMGB1 knockdown was verified in SGC cells through qRT-PCR assay. (b) The efficiency of HMGB1 overexpression was verified in HGC cells through qRT-PCR assay. The expression levels of HMGB1 protein in the nucleus and cytoplasm of SEMA3B-AS1 (c) overexpressed or (d) interfered cells were detected by Western blotting. (e) The localization of HMGB1 was analyzed by immunofluorescence when SEMA3B-AS1 was overexpressed. Red fluorescence represents HMGB1. (f) The localization of HMGB1 was analyzed by immunofluorescence when SEMA3B-AS1 was knocked down. Red fluorescence represents HMGB1. (g) Dual-Luciferase Assays to assess FBXW7 promoter activity while SEMA3B-AS1 was either alone or in combination with HMGB1. (h) The changes of FBXW7 protein were detected by Western blotting after overexpression or interference of HMGB1 in SEMA3B-AS1-overexpressed gastric cancer cells.

glycosylation, and acetylation. We speculated that the BGN protein is degraded by ubiquitination. Then, we were surprised to find that the BGN protein is the ubiquitination substrate of FBXW7 through UbiBrowser website prediction. To verify our speculation, the mRNA expression of FBXW7 was detected by qRT-PCR in 5 gastric cancer cell lines (AGS, HGC, MGC, MKN, and SGC) and GES-1
(Figure 5(d)). According to the results, SGC was selected as the knockdown cell line, and HGC was selected as the overexpression cell line. The overexpression or knockdown efficiency of FBXW7 was verified through qRT-PCR assay (Figures 5(e) and 5(f)). To demonstrate that FBXW7 regulates BGN at the posttranscriptional level, BGN mRNA and protein expression levels were tested in FBXW7- 

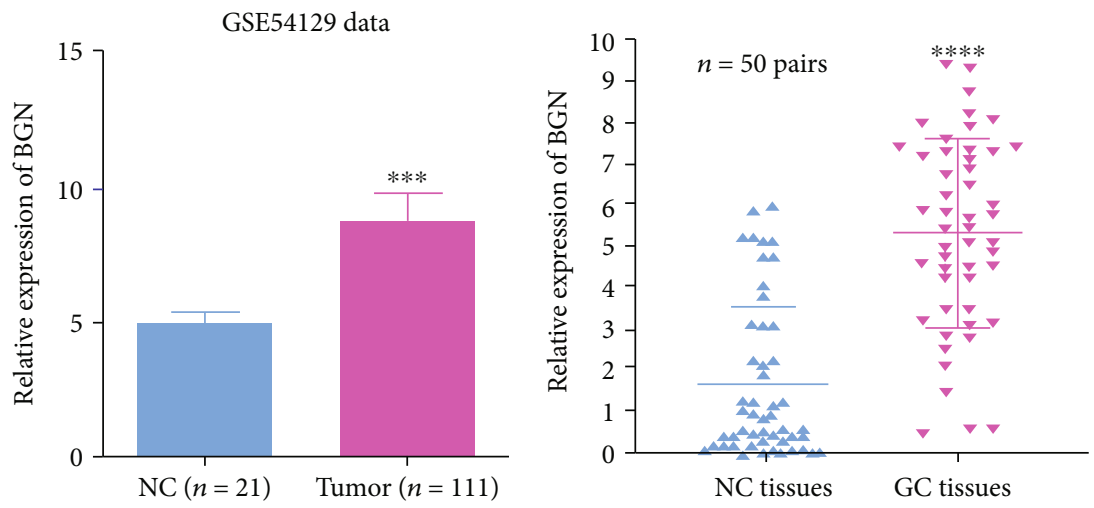

(a)

(b)

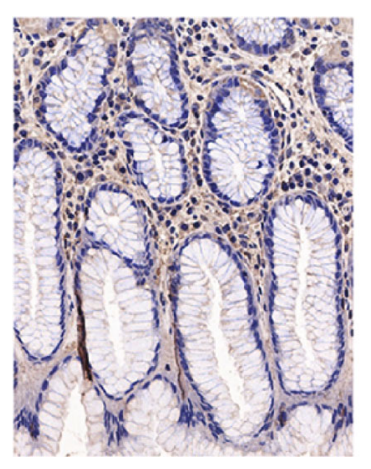

BGN-NC

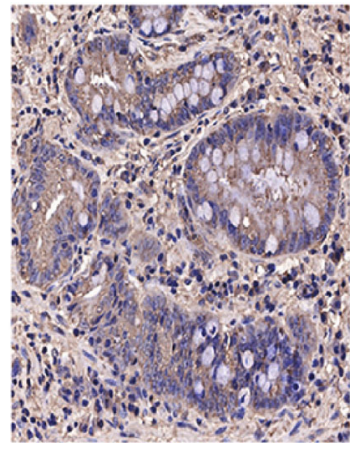

BGN-GC

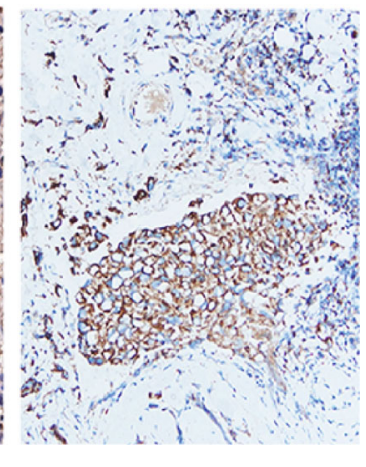

(c)

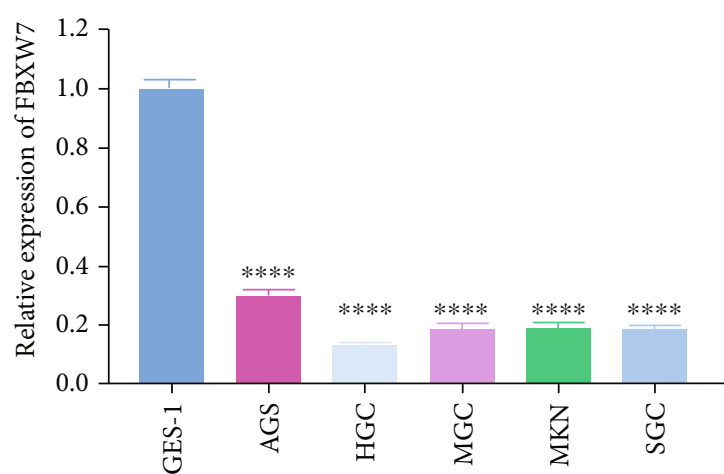

(d)

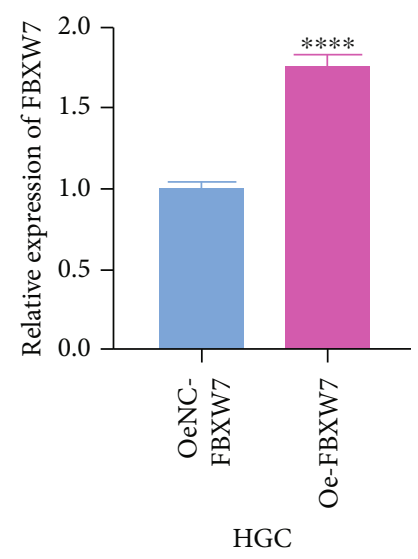

(e)

FIgUre 5: Continued. 


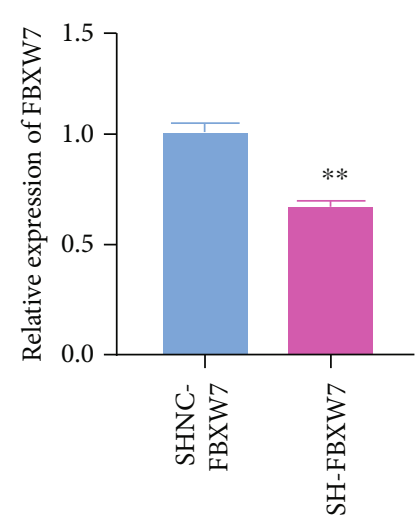

(f)

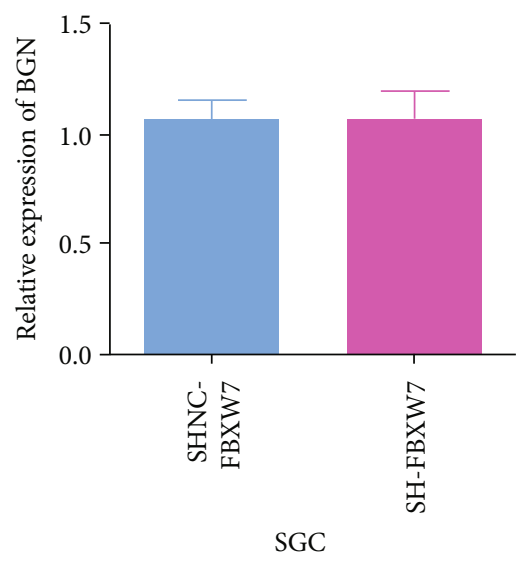

(h)

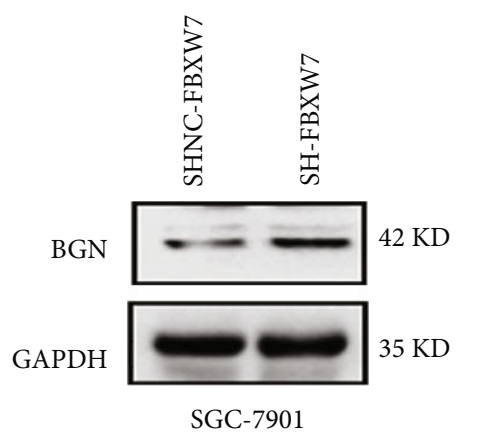

(j)

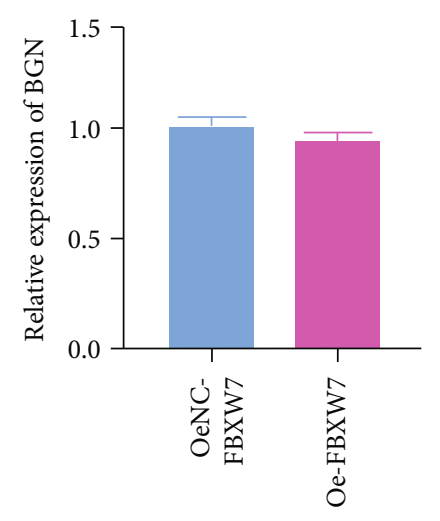

HGC

(g)

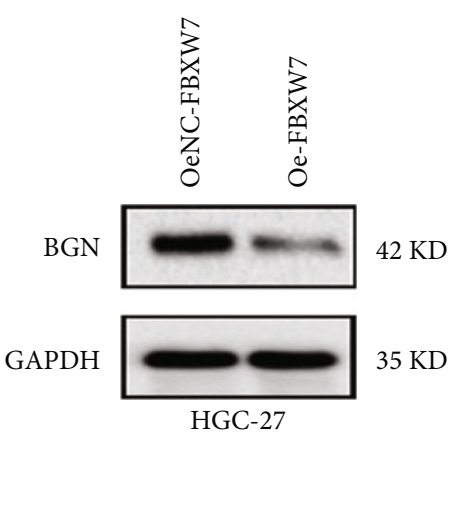

(i)

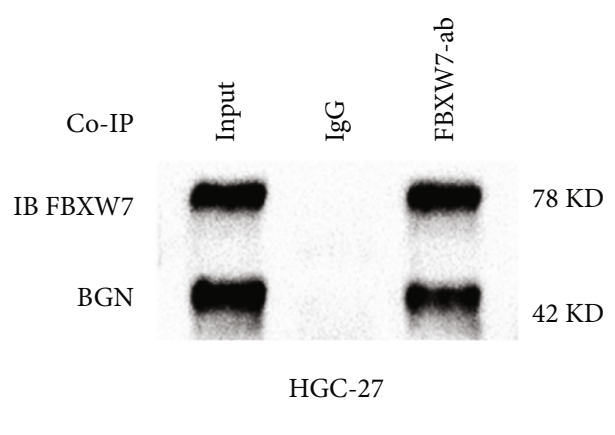

(k)

FIgUre 5: Continued. 


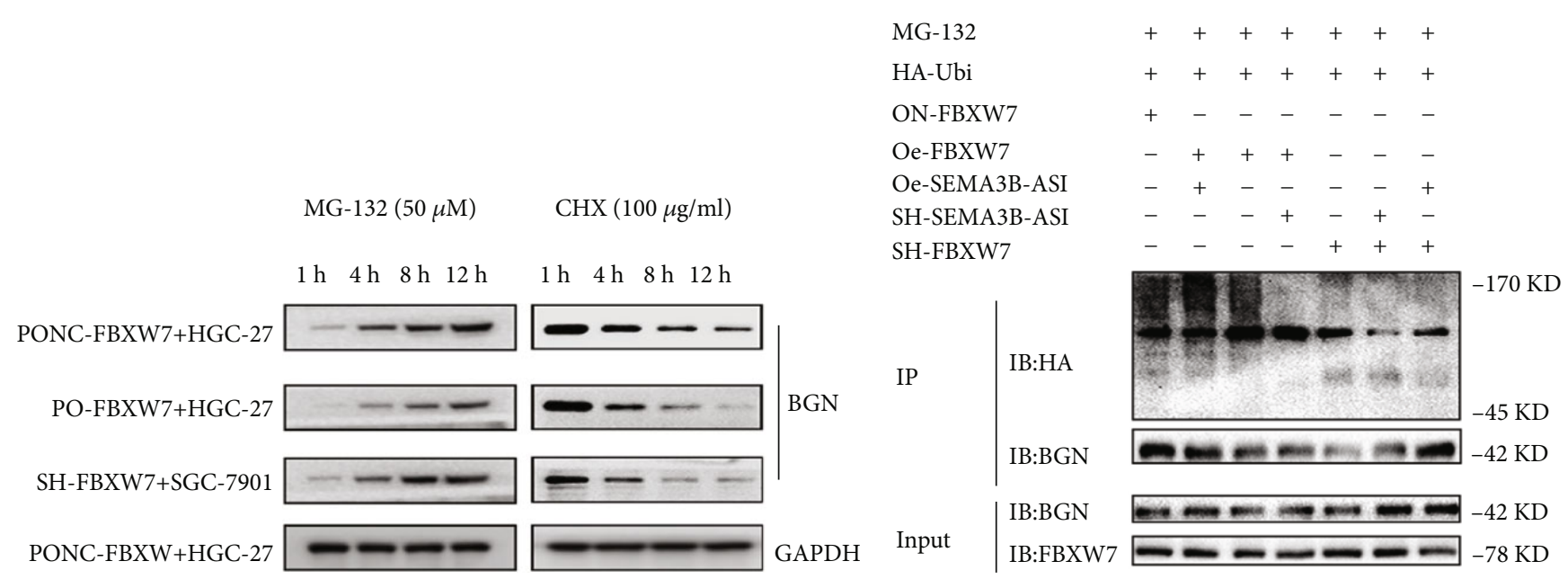

(l)

$(\mathrm{m})$

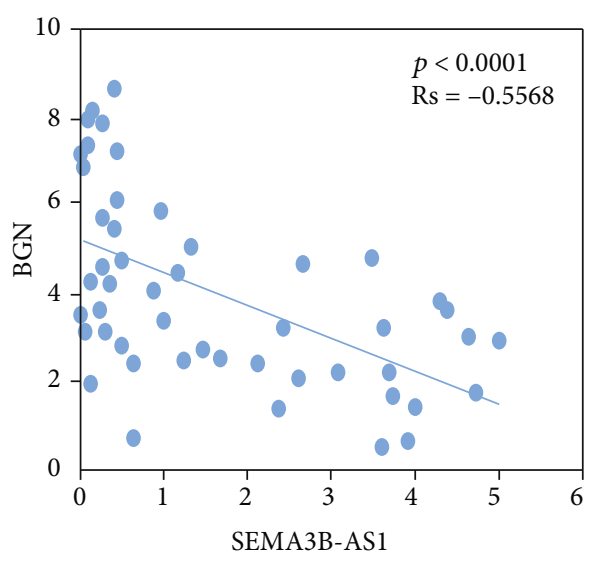

(n)

FIGURE 5: SEMA3B-AS1 might destabilize the BGN protein by regulating FBXW7, thus promoting the ubiquitination degradation of BGN and inhibiting GC. Data were shown as mean \pm SEM $(n=3) ;{ }^{*} p<0.05,{ }^{* *} p<0.01,{ }^{* * *} p<0.001$, and ${ }^{* * * *} p<0.0001$. (a) The expression of BGN protein in gastric cancer and adjacent normal tissues was analyzed by bioinformatics using GSE54129 data set. (b) The relative mRNA expression of BGN was identified by qRT-PCR assay in 50 pairs of GC tissues and adjacent normal tissues. (c) The representative IHC staining image for BGN in the paracancerous normal tissues, GC tissues, and GS PM tissues. (d) The relative mRNA expression of FBXW7 in five GC cell lines and normal stomach epithelial cell line (GES-1) was detected by qRT-PCR assay. (e) The overexpression efficiency of FBXW7 and negative control (NC) was detected in HGC-27 using qRT-PCR. (f) The knockdown efficiency of FBXW7 and negative control (NC) was detected in SGC-7901 using qRT-PCR. The mRNA expression levels of BGN were detected by qRT-PCR assay after FBXW7 (g) overexpression or (h) knockdown in GC cells (HGC-27 or SGC-7901). The protein expression levels of BGN were detected by Western blotting assay after FBXW7 (i) overexpression or (j) knockdown in GC cells (HGC-27 or SGC-7901). (k) The interaction between FBXW7 and BGN was identified in HGC-27 cells by coimmunoprecipitation (co-IP). SDS-PAGE separated the immunoprecipitates of input (20\%) and BGN. Western blotting was performed to confirm the interaction between FBXW7 and BGN. (l) Cell groups were treated with the proteasome inhibitor MG-132 $(50 \mu \mathrm{M})$ or the protein synthesis inhibitor cycloheximide CHX (100 $\mu \mathrm{g} /$ $\mathrm{mL}$ ) at different time points $(1 \mathrm{~h}, 4 \mathrm{~h}, 8 \mathrm{~h}$, and $12 \mathrm{~h})$, and the changes of BGN protein at different time points were detected by Western blotting assay. (m) HGC-27 or SGC-7901 cells were treated with MG-132 (50 $\mathrm{MM})$ and transfected with HA-Ubi and simultaneously overexpression or knockdown FBXW7 as well as SEMA3B-AS1 overexpressed or knocked down, respectively, FBXW7 vector served as a negative control. Cell lysates were immunoprecipitated with anti-BGN antibody to identify ubiquitination of BGN with anti-HA antibodies using Western blotting assay. (n) The mRNA relative expression levels of SEMA3B-AS1 and BGN were obvious negative association in GC patients using Pearson's correlation analysis ( $\mathrm{Rs}=-0.5568, p<0.0001)$.

overexpressing (HGC-27) or FBXW7 knockdown (SGC7901) GC cells by qRT-PCR and Western blotting assays. The results showed that the BGN mRNA expression level was not significantly affected by FBXW7 overexpression or knockdown (Figures 5(g) and 5(h)), but the BGN protein level was significantly decreased or increased with FBXW7 overexpression or knockdown (Figures 5(i) and 5(j)). Next, we needed to prove the interaction between FBXW7 and BGN proteins, so a co-IP assay was performed, and the results demonstrated this fact (Figure 5(k)). Furthermore, to further test whether BGN is degraded by the ubiquitination pathway, we treated Lv-Oe-FBXW7-HGC-27 and LvOeNC-FBXW7-HGC-27 cells with cycloheximide (CHX) (a protein synthesis inhibitor) and MG-132 (a specific 
proteasome inhibitor). We found that MG-132 abolished the downregulation of BGN protein levels in FBXW7overexpressing cells (Figure 5(1)). These results indicated that the ubiquitin-proteasome pathway might be required for the FBXW7-mediated degradation of BGN protein. To confirm that the ubiquitination level of BGN was affected by FBXW7, we conducted an IP assay and ubiquitination experiment after GC cell treatment with MG-132 $(50 \mu \mathrm{M})$, HA-Ubi plasmid transfection and FBXW7 overexpression or knockdown. The ubiquitination level of BGN was assessed with anti-HA Tag antibody. The ubiquitination level of BGN was increased in the FBXW7 overexpression group compared with the negative control group. However, the ubiquitination level of BGN was decreased after FBXW7 knockdown (Figure 5(m)). Moreover, we found a negative regulatory relationship between SEMA3B-AS1 and BGN by tissue validation of SEMA3B-AS1 and BGN $(p<0.0001)$ (Figure 5(n)). Further experiments have shown that when we overexpressed FBXW7 and SEMA3B-AS1 in HGC cells, the BGN ubiquitination level was the highest, and when we knocked down both factors in SGC cells, the BGN ubiquitination level was the lowest. However, when we simultaneously knocked down or overexpressed SEMA3B-AS1 in FBXW7-overexpressing or knockdown cells, the changes in BGN ubiquitination levels were partially reversed (Figure 5(m)). In conclusion, we found that FBXW7 could ubiquitylate the BGN protein, and its function was dependent on SEMA3B-AS1 to a large extent. At the same time, we could prove that the SEMA3B-AS1/HMGB1/FBXW7 axis exists.

3.7. Animal Experiments. We further explored the role of SEMA3B-AS1 in the oncogenesis and metastasis of GC. We conducted animal experiments. For the subcutaneous xenograft model, six- to eight-week-old nude mice were randomly divided into three groups $(n=5)$, and OeNCSEMA3B-AS1-HGC cells $\left(5 \times 10^{5}\right)$, Oe-SEMA3B-AS1HGC cells $\left(5 \times 10^{5}\right)$, and Oe-SEMA3B-AS1+SH-FBXW7HGC cells $\left(5 \times 10^{5}\right)$ in $0.1 \mathrm{~mL}$ DMEM were injected into the flanks of mice. After 2 weeks, we began to measure the tumor size every week using digital vernier calipers and calculated the tumor volume using the following formula: volume $=1 / 2 \times\left(\right.$ width $^{2} \times$ length $)$. At the fifth week of cell injection, the mice were euthanized, and subcutaneous tumors were removed. The results indicated that the volume and weight of tumors in the Oe-SEMA3B-AS1 group were significantly smaller than those in the OeNC-SEMA3BAS1-HGC cell group and the Oe-SEMA3B-AS1+SHFBXW7-HGC cell group (Figures 6(a)-6(c)). Furthermore, we performed an immunohistochemistry assay to identify the protein expression of FBXW7and BGN in the three groups of tumors. The results demonstrated that the expression of FBXW7 was significantly increased in the OeSEMA3B-AS1 group, while the BGN protein expression level was dramatically decreased (Figure 6(d)). For the intraperitoneal tumor formation model, Oe-SEMA3B-AS1-HGC cells $\left(1 \times 10^{6}\right)$,Oe-SEMA3B-AS1-HGC cells $\left(1 \times 10^{6}\right)$, and Oe-SEMA3B-AS1+SH-FBXW7-HGC cells $\left(1 \times 10^{6}\right)$ were injected into the lower right abdominal cavity of each mouse. After 35 days, we euthanized the mice and dissected the abdominal cavity to assess tumor formation. We assessed abdominal invasion, including PM (1/6 mice), perigastric metastasis (0/6 mice), intestinal and mesenteric metastasis (3/6 mice), and diaphragmatic metastasis (0/6 mice), after SEMA3B-AS1 overexpression. However, in theOeNC-SEMA3B-AS1 group, we observed PM (4/6 mice), perigastric metastasis (4/6 mice), intestinal and mesenteric metastasis (5/6 mice), and diaphragmatic metastasis $(2 / 6$ mice). Statistical analysis of the two groups of data showed that the difference was statistically significant $(p=0.027)$. When we knocked down FBXW7 in the OE-SEMA3B-AS1 group, the tumor suppressive effect of SEMA3B-AS1 was partially attenuated (Figures 6(e) and 6(f)). These data demonstrated that SEMA3B-AS1 can inhibit GC oncogenesis in vivo. Taken together, these findings indicate that SEMA3B-AS1 can regulate GC formation and PM by regulating the BGN protein. The summary schematic of our findings from the current study is shown in Figure 6(g).

\section{Discussion}

Nuclear lncRNAs play a crucial role in many biological processes, and their dysregulation can lead to a variety of human cancers, including GC $[29,30]$. Our present study is conducive to understanding the role of SEMA3B-AS1 downregulation in GC progression. Our data demonstrated that SEMA3B-AS1 overexpression could inhibit the PM of GC by regulating BGN protein ubiquitination. Some studies have found that SEMA3B-AS1 is an antioncogene in various cancers, including gastric cardia adenocarcinoma and esophageal squamous cell carcinoma [31, 32]. In these studies, SEMA3B-AS1 was confirmed to inhibit the progression of cardiac adenocarcinoma and to be closely related to the progression and prognosis of esophageal squamous cell carcinoma. Our data also confirmed that SEMA3B-AS1 plays an important role in GC progression and PM and is closely related to the survival and prognosis of GC patients.

HMGB1 plays a complex role in a variety of biological processes and has a variety of functions in maintaining cell homeostasis, and it is involved in the development of many diseases, such as inflammation and cancer. Recent studies have indicated that its function is closely related to its localization. It mainly assists in regulating telomeres and maintaining genomic stability in the nucleus, and its loss can lead to genomic instability and tumorigenesis [15, 16]. In the present study, we found that HMGB1 and SEMA3BAS1 obviously interacted through RNA pull-down experiments when SEMA3B-AS1 was overexpressed. Moreover, we also demonstrated using RIP and ChIRP experiments that SEMA3B-AS1 can bind to HMGB1, thereby playing an important biological role. Furthermore, immunofluorescence localization revealed that HMGB1 was mainly located in the nucleus during SEMA3B-AS1 overexpression. However, after SEMA3B-AS1 interference, the HMGB1 was mainly located in the cytoplasm. Our experiments revealed that SEMA3B-AS1 can recruit and bind HMGB1 in the nucleus. In our previous studies, we demonstrated that 


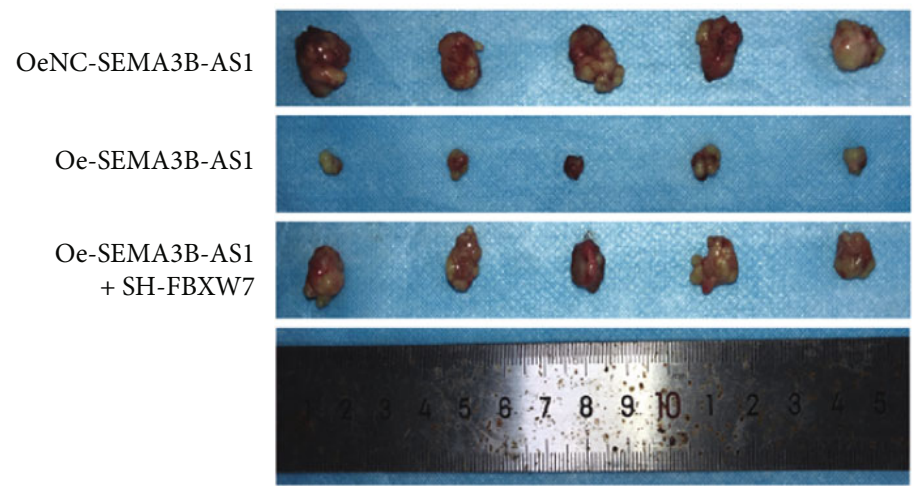

(a)

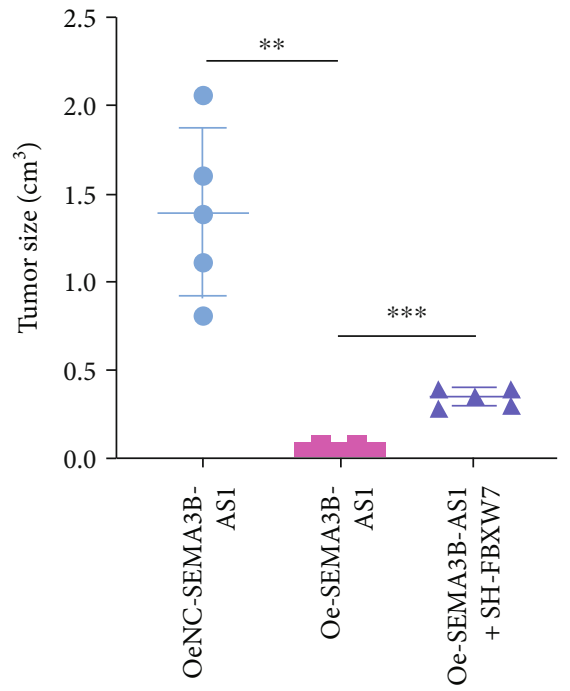

(b)

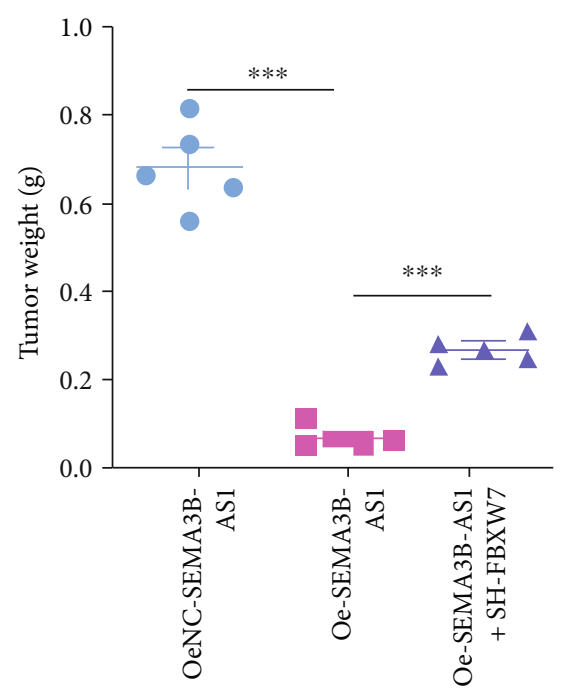

(c)

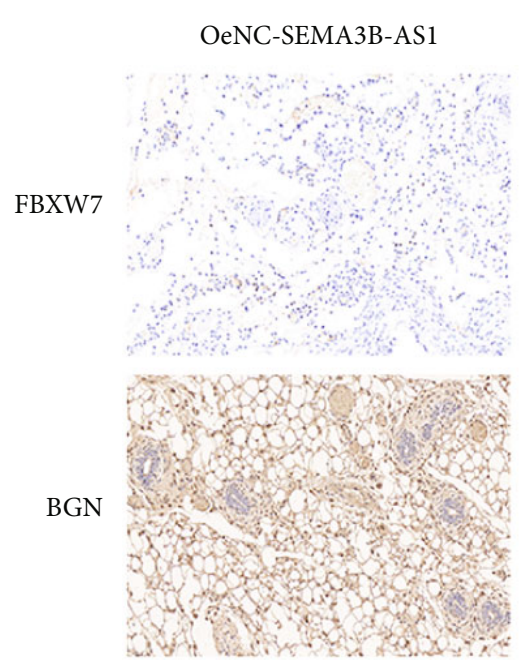

Oe-SEMA3B-AS1

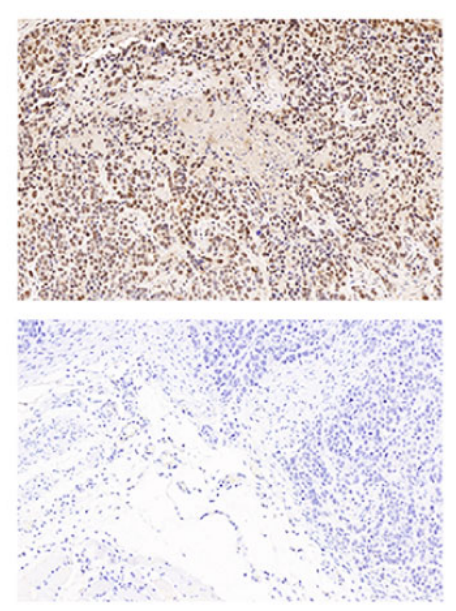

Oe-SEMA3B-AS1

+ SH-FBXW7

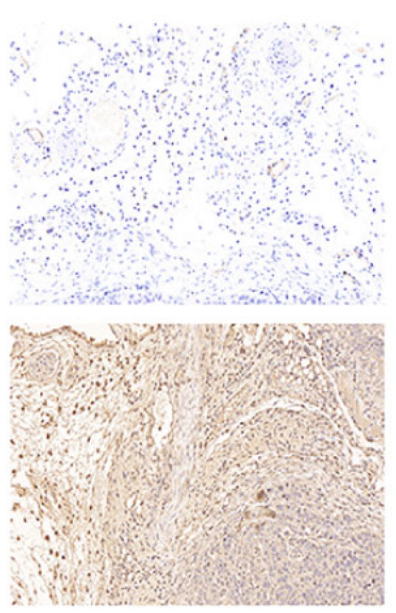

(d)

Figure 6: Continued. 


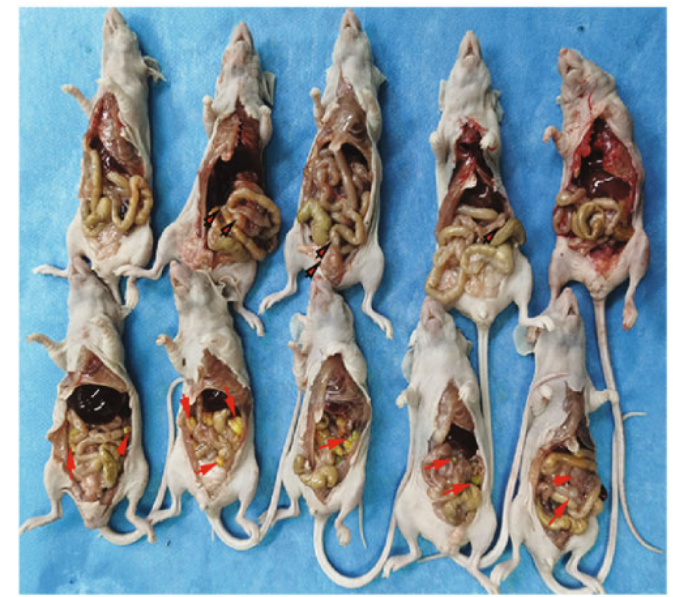

(e)

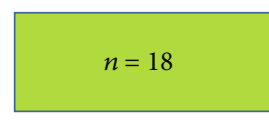

Peritoneal
metastasis
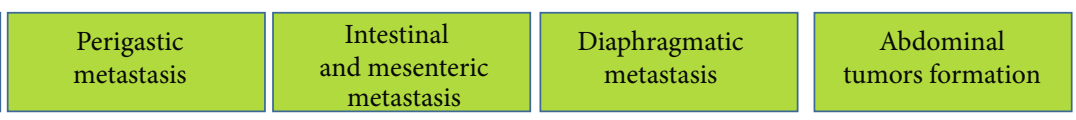

OeNC-SEMA3B-
AS1 $(n=6)$
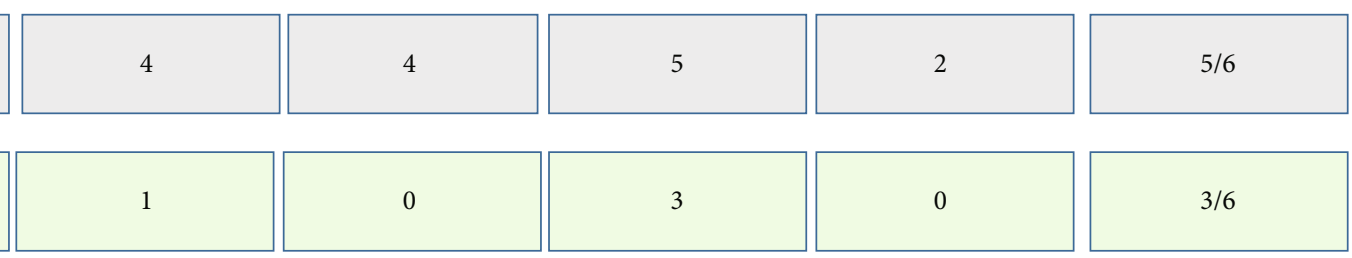

\begin{tabular}{c}
$\begin{array}{c}\text { Oe-SEMA3B- } \\
\text { AS1 }(n=6)\end{array}$ \\
\hline
\end{tabular}
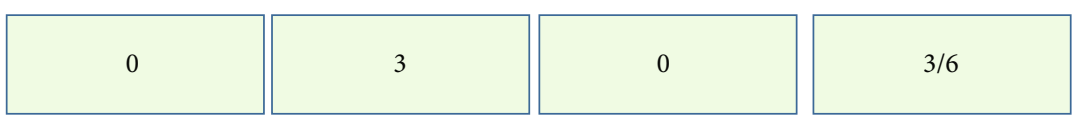

\begin{tabular}{c} 
Oe-SEMA3B- \\
AS1+SH-FBXW7 \\
$(n=6)$ \\
\hline
\end{tabular}
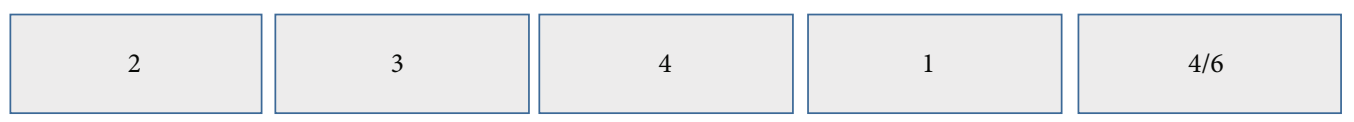

(f)

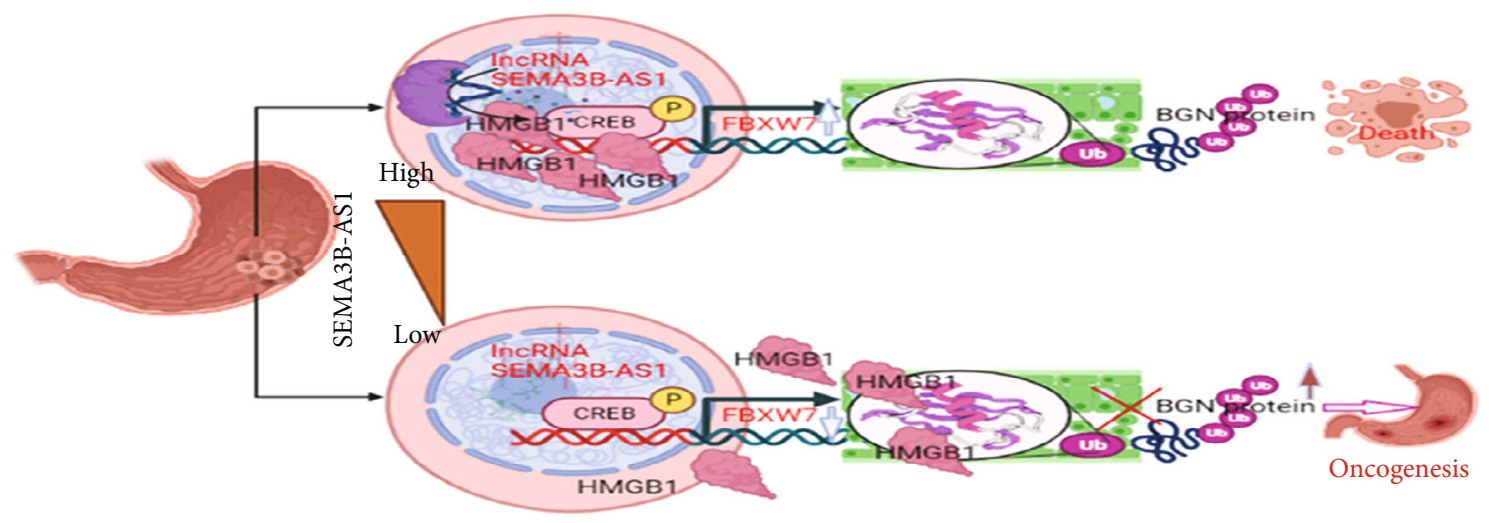

(g)

Figure 6: SEMA3B-AS1 could inhibit GC tumorigenesis and PM in vivo. Representative images of migratory or invaded cells (magnification, $\times 200$ ) were shown. Data are shown as mean \pm SEM $(n=3) ;{ }^{*} p<0.05,{ }^{* *} p<0.01$, and ${ }^{* * *} p<0.001$. The morphological properties of (a) tumor subcutaneous xenograft, (b) tumor size, and (c) tumor weight in OeNC-SEMA3B-AS1-HGC-27, Oe-SEMA3BAS1, and Oe-SEMA3B-AS1+SH-FBXW7-HGC-27 cells at 35 days, each group had five mice. (d) IHC analyzed the expression of FBXW7 and BGN protein of tumors from the OeNC-SEMA3B-AS1-HGC-27, Oe-SEMA3B-AS1, and Oe-SEMA3B-AS1+SH-FBXW7-HGC-27 cell groups. (e) The representative image of intraperitoneal tumor formation model from OeNC-SEMA3B-AS1-HGC-27 and OeSEMA3B-AS1-HGC-27. (f) Simultaneously, we counted and analyzed the number of peritoneal, perigastric, intestinal and mesenteric, and diaphragmatic metastases, each group had six mice. (g) The proposed mechanism model in which the SEMA3B-AS1/HMGB1/ FBXW7 axis mediates the PM of GC by regulating BGN protein ubiquitination.

FBXW7 can inhibit the progression and predict the prognosis of GC $[19,28]$. In the current research, we found that the tumor suppressor gene FBXW7 is usually mutated and inac- tivated after the occurrence of cancer, and the body sends instructions for gene repair, which allows the antioncogene lncRNA to recruit and bind genes or proteins that can repair 
DNA damage. According to our data, we demonstrated that when SEMA3B-AS1 is overexpressed, HMGB1 is recruited. Then, SEMA3B-AS1 can promote the transcription and expression of FBXW7 through binding HMGB1, thus promoting autophagy and inhibiting apoptosis in tumor cells. In the absence of cancer-suppressive lncRNAs such as SEMA3B-AS1, HMGB1 cannot be recruited to play a vital role in DNA repair in time, but it may be transferred to the cytoplasm and extracellular space. The location of HMGB1 in the cytoplasm or extracellular space will promote the release of cytokines such as IL- 6 and IL- 8 by activating MAPK- and MyD88-dependent NF-KB pathways, thereby prompting tumor cell proliferation, angiogenesis, EMT, invasion, and metastasis. We revealed for the first time that SEMA3B-AS1 can bind HMGB1 to promote the transcription and expression of FBXW7, thus exerting its tumor suppressive effect.

In our analysis of MS results, we found that BGN protein expression was abnormally upregulated in PM samples. Recently, some studies have shown that abnormal expression of the BGN protein is closely associated with tumor metastasis and a poor prognosis for cancers, including GC and breast cancer $[23,33]$. However, there are few studies on the degradation pathway of the BGN protein. Further exploration of BGN metabolism will provide more favorable evidence for the diagnosis and treatment of the PM of GC in the future. Existing research indicates that the ubiquitinproteasome pathway is a very important factor in the regulation of tumor immunity, tumorigenesis, development, and prognosis. This is a vital pathway for protein degradation in eukaryotic cells, and approximately $80 \%-90 \%$ of proteins involved in cell function are degraded through the ubiquitin-proteasome pathway [20]. Our studies further indicated that the BGN protein could be ubiquitinated by FBXW7. These discoveries provide a theoretical basis for the prognostic evaluation, diagnosis, and treatment of PM in GC patients.

\section{Conclusions}

In summary, SEMA3B-AS1 inhibits the PM of GC by regulating the expression of the BGN protein. The specific mechanism is that SEMA3B-AS1 promotes the transcription and expression of FBXW7 by binding HMGB1 in the nucleus, and FBXW7 degrades the BGN protein via the ubiquitinproteasome pathway. Therefore, SEMA3B-AS1 may be a novel and promising therapeutic target and biological marker for GC patients with PM.

$\begin{array}{ll}\text { Abbreviations } & \\ \text { GC: } & \text { Gastric cancer } \\ \text { BGN: } & \text { Biglycan } \\ \text { EMT: } & \text { Epithelial-mesenchymal transition } \\ \text { SEMA3B-AS1: } & \text { Long noncoding RNA SEMA3B-AS1 } \\ \text { NC: } & \text { Negative control } \\ \text { qRT-PCR: } & \text { Quantitative reverse transcription } \\ & \text { polymerase chain reaction } \\ \text { PM: } & \text { Peritoneal metastasis }\end{array}$

PMT:

LNM:

TNM:

DM:

CA19-9:

CEA:

IHC:

IP:

CO-IP:

RIP:

ChIP:

ChIRP:

Lv-Oe-SEMA3B-

AS1:

Lv-ON-SEMA3B-

AS1:

PO-SEMA3B-AS1:

PN-SEMA3B-AS1:

Lv-SH-SEMA3B-

AS1:

Lv-SHNC-SEMA3B-

AS1:

FBXW7:

Lv-Oe-FBXW7:

Lv-ON-FBXW7:

SH-FBXW7:

SHNC-FBXW7:

HMGB1:

\section{Data Availability}

The data used to support the findings of this study are available from the corresponding author upon request.

\section{Conflicts of Interest}

All authors declare no conflict of interest.

\section{Authors' Contributions}

Guoquan Huang, Zhenxian Xiang, Shuyi Wang, Chaogang Yang, and Bin Xiong were responsible for the research design. Guoquan Huang, Zhenxian Xiang, Haitao Wu, and Bin Xiong were responsible for data analysis. Guoquan Huang, Zhenxian Xiang, Haitao Wu, Qiuming He, Rongzhang Dou, and Sihao Huang performed the experiment. Guoquan Huang, Zhenxian Xiang, Haitao Wu, and Qiuming He wrote or contributed to the writing of the manuscript. Guoquan Huang, Zhenxian Xiang, and Haitao Wu contributed equally to this work.

\author{
Peritoneal metastasis tissue \\ ymph node metastasis \\ -node-metastasis \\ Carbohydrate antigen 19-9 \\ moprecipitation \\ RNA-binding protein \\ Chromatin immunoprecipitation \\ cation chromatin isolation by RNA \\ Lentivirus-mediated overexpression \\ of SEMA3B-AS1 \\ of SEMA3B-AS1 \\ AS1 \\ pCDNA-negative control of \\ Lentivirus-mediated knockdown of \\ SEMA3B-AS1 \\ of SEMA3B-AS1 \\ F-box and WD repeat domain- \\ Lentivirus-mediated overexpression \\ of FBXW7 \\ pCDNA-knockdown negative control \\ of FBXW7 \\ High mobility group box 1 .
}




\section{Acknowledgments}

Thanks are due to Hubei Provincial Cancer Clinical Research Center for providing us with the experimental platform. Our study was funded by grants from the National Natural Science Foundation of China (81872376), the National Natural Science Fund Youth Fund of China (81702411), the Health Commission of Hubei Province Scientific Research Project (WJ2019H012), and the Clinical Special Disease Diagnosis and Treatment Technology Foundation of Zhongnan Hospital of Wuhan University (ZLYNXM202018).

\section{Supplementary Materials}

Supplementary 1. Supplementary Table 1: related gene primers mentioned in this article.

Supplementary 2. Supplementary Table 2: annotation information for RNA pull-down mass spectrometry.

\section{References}

[1] R. L. Siegel, K. D. Miller, and A. Jemal, "Cancer statistics, 2015," CA: a Cancer Journal for Clinicians, vol. 65, no. 1, pp. 5-29, 2015.

[2] W. Chen, R. Zheng, P. D. Baade et al., "Cancer statistics in China, 2015," CA: a Cancer Journal for Clinicians, vol. 66, no. 2, pp. 115-132, 2016.

[3] H. Wilke, K. Muro, E. van Cutsem et al., "Ramucirumab plus paclitaxel versus placebo plus paclitaxel in patients with previously treated advanced gastric or gastro-oesophageal junction adenocarcinoma (RAINBOW): a double-blind, randomised phase 3 trial," The Lancet Oncology, vol. 15, no. 11, pp. 1224-1235, 2014.

[4] Y.-J. Bang, E. van Cutsem, A. Feyereislova et al., "Trastuzumab in combination with chemotherapy versus chemotherapy alone for treatment of HER2-positive advanced gastric or gastro-oesophageal junction cancer (ToGA): a phase 3, openlabel, randomised controlled trial," The Lancet, vol. 376, no. 9742, pp. 687-697, 2010.

[5] F. Coccolini, E. Cotte, O. Glehen et al., "Intraperitoneal chemotherapy in advanced gastric cancer. Meta-analysis of randomized trials," European Journal of Surgical Oncology, vol. 40, no. 1, pp. 12-26, 2014.

[6] D. Dong, L. Tang, Z. Y. Li et al., "Development and validation of an individualized nomogram to identify occult peritoneal metastasis in patients with advanced gastric cancer," Annals of Oncology, vol. 30, no. 3, pp. 431-438, 2019.

[7] K. Fujitani, H.-K. Yang, J. Mizusawa et al., "Gastrectomy plus chemotherapy versus chemotherapy alone for advanced gastric cancer with a single non-curable factor (REGATTA): a phase 3, randomised controlled trial," The Lancet Oncology, vol. 17, no. 3, pp. 309-318, 2016.

[8] J. T. Lee, "Epigenetic regulation by long noncoding RNAs," Science, vol. 338, no. 6113, pp. 1435-1439, 2012.

[9] C. P. Ponting, P. L. Oliver, and W. Reik, "Evolution and functions of long noncoding RNAs," Cell, vol. 136, no. 4, pp. 629641, 2009.

[10] K. C. Wang and H. Y. Chang, "Molecular mechanisms of long noncoding RNAs," Molecular Cell, vol. 43, no. 6, pp. 904-914, 2011.
[11] X. Hu, Y. Feng, D. Zhang et al., "A functional genomic approach identifies_FAL1_ as an oncogenic long noncoding RNA that associates with BMI1 and represses p21 expression in cancer," Cancer Cell, vol. 26, no. 3, pp. 344357, 2014.

[12] T. R. Mercer, M. E. Dinger, and J. S. Mattick, "Long noncoding RNAs: insights into functions," Nature Reviews. Genetics, vol. 10, no. 3, pp. 155-159, 2009.

[13] J. E. Wilusz, H. Sunwoo, and D. L. Spector, "Long noncoding RNAs: functional surprises from the RNA world," Genes \& Development, vol. 23, no. 13, pp. 1494-1504, 2009.

[14] X. Huang, R. Xiao, S. Pan et al., "Uncovering the roles of long non-coding RNAs in cancer stem cells," Journal of Hematology \& Oncology, vol. 10, no. 1, p. 62, 2017.

[15] S. Yuan, Z. Liu, Z. Xu, J. Liu, and J. Zhang, "High mobility group box 1 (HMGB1): a pivotal regulator of hematopoietic malignancies," Journal of Hematology \& Oncology, vol. 13, no. 1, p. 91, 2020.

[16] Y. Yang, L. Yang, S. Jiang et al., "HMGB1 mediates lipopolysaccharide-induced inflammation via interacting with GPX4 in colon cancer cells," Cancer Cell International, vol. 20, no. 1, p. 205, 2020.

[17] M. M. Lou, X. Q. Tang, G. M. Wang et al., "Long noncoding RNA BS-DRL1 modulates the DNA damage response and genome stability by interacting with HMGB1 in neurons," Nature Communications, vol. 12, no. 1, p. 4075, 2021.

[18] W. Ni, S. Yao, Y. Zhou et al., "Long noncoding RNA GAS5 inhibits progression of colorectal cancer by interacting with and triggering YAP phosphorylation and degradation and is negatively regulated by the $\mathrm{m6A}$ reader YTHDF3," Molecular Cancer, vol. 18, no. 1, p. 143, 2019.

[19] L. I. Hui, C. Zhang, W. U. Quanfeng, and J. Sun, "The expressions of CDC4 and c-Myc in gastric cancer and their clinical signifieance," China Oncology, vol. 25, pp. 933-939, 2015.

[20] D. Chen and Q. Ping Dou, “The ubiquitin-proteasome system as a prospective molecular target for cancer treatment and prevention," Current Protein \& Peptide Science, vol. 11, no. 6, pp. 459-470, 2010.

[21] J. R. Fallon and E. M. McNally, "Non-glycanated biglycan and LTBP4: leveraging the extracellular matrix for Duchenne muscular dystrophy therapeutics," Matrix Biology, vol. 68-69, pp. 616-627, 2018.

[22] D. Guo, W. Zhang, H. Yang et al., "Celastrol induces necroptosis and ameliorates inflammation via targeting biglycan in human gastric carcinoma," International Journal of Molecular Sciences, vol. 20, no. 22, p. 5716, 2019.

[23] H. C. Huang, B. H. Cai, C. S. Suen et al., "BGN/TLR4/NF-B mediates epigenetic silencing of immunosuppressive Siglec ligands in colon cancer cells," Cell, vol. 9, no. 2, p. 397, 2020.

[24] E. Wiechec, M. Magan, N. Matic et al., "Cancer-associated fibroblasts modulate transcriptional signatures involved in proliferation, differentiation and metastasis in head and neck squamous cell carcinoma," Cancers, vol. 13, no. 13, p. 3361, 2021.

[25] J. R. Egner, “AJCC Cancer Staging Manual,” JAMA, vol. 304, no. 15, pp. 1726-1727, 2010.

[26] K. Berns, H. M. Horlings, B. T. Hennessy et al., "A functional genetic approach identifies the PI3K pathway as a major determinant of trastuzumab resistance in breast cancer," Cancer Cell, vol. 12, no. 4, pp. 395-402, 2007. 
[27] F. Kopp and J. T. Mendell, "Functional classification and experimental dissection of long noncoding RNAs," Cell, vol. 172, no. 3, pp. 393-407, 2018.

[28] H. Li, Z. Wang, W. Zhang, K. Qian, W. Xu, and S. Zhang, "Fbxw7 regulates tumor apoptosis, growth arrest and the epithelial-to- mesenchymal transition in part through the RhoA signaling pathway in gastric cancer," Cancer Letters, vol. 370, no. 1, pp. 39-55, 2016.

[29] M. Ma, Y. Zhang, M. Weng et al., "IncRNA GCAWKR promotes gastric cancer development by scaffolding the chromatin modification factors WDR5 and KAT2A," Molecular Therapy, vol. 26, no. 11, pp. 2658-2668, 2018.

[30] P. Qi and X. Du, "The long non-coding RNAs, a new cancer diagnostic and therapeutic gold mine," Modern Pathology, vol. 26, no. 2, pp. 155-165, 2013.

[31] Z. Dong, X. Liang, X. Wu et al., "Promoter hypermethylationmediated downregulation of tumor suppressor gene SEMA3B and lncRNA SEMA3B-AS1 correlates with progression and prognosis of esophageal squamous cell carcinoma," Clinical \& Experimental Metastasis, vol. 36, no. 3, pp. 225-241, 2019.

[32] W. Guo, X. Liang, L. Liu et al., "MiR-6872 host gene SEMA3B and its antisense lncRNA SEMA3B-AS1 function synergistically to suppress gastric cardia adenocarcinoma progression," Gastric Cancer, vol. 22, no. 4, pp. 705-722, 2019.

[33] Breast Cancer Association Consortium, L. Dorling, S. Carvalho et al., "Breast Cancer Risk Genes - Association Analysis in More than 113,000 Women," The New England Journal of Medicine, vol. 384, no. 5, pp. 428-439, 2021. 